ARTICLE

\title{
Dysregulation of mitochondria-lysosome contacts by GBA1 dysfunction in dopaminergic neuronal models of Parkinson's disease
}

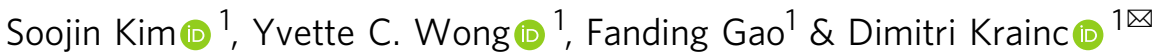

Mitochondria-lysosome contacts are recently identified sites for mediating crosstalk between both organelles, but their role in normal and diseased human neurons remains unknown. In this study, we demonstrate that mitochondria-lysosome contacts can dynamically form in the soma, axons, and dendrites of human neurons, allowing for their bidirectional crosstalk. Parkinson's disease patient derived neurons harboring mutant GBA1 exhibited prolonged mitochondria-lysosome contacts due to defective modulation of the untethering protein TBC1D15, which mediates Rab7 GTP hydrolysis for contact untethering. This dysregulation was due to decreased GBA1 ( $\beta$-glucocerebrosidase (GCase)) lysosomal enzyme activity in patient derived neurons, and could be rescued by increasing enzyme activity with a GCase modulator. These defects resulted in disrupted mitochondrial distribution and function, and could be further rescued by TBC1D15 in Parkinson's patient derived GBA1-linked neurons. Together, our work demonstrates a potential role of mitochondria-lysosome contacts as an upstream regulator of mitochondrial function and dynamics in midbrain dopaminergic neurons in GBA1-linked Parkinson's disease.

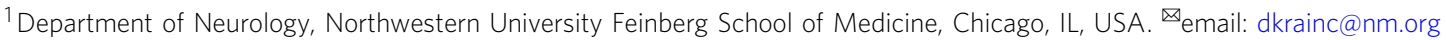


M itochondria-lysosome (M-L) contact sites were recently identified as inter-organelle membrane contacts involving the dynamic tethering of mitochondria with lysosomes ${ }^{1,2}$. Importantly, M-L contacts allow for the bidirectional regulation of both mitochondrial and lysosomal network dynamics, and mediate their direct interaction in a pathway distinct from mitophagy or lysosomal degradation of mitochondrial-derived vesicles ${ }^{2}$. M-L contact sites are further regulated by TBC1D15, a Rab7 GAP recruited to mitochondria via the outer mitochondrial membrane protein Fis $1^{3-6}$, which mediates Rab7 GTP hydrolysis to promote M-L contact untethering dynamics ${ }^{2}$. However, the formation and role of $\mathrm{M}-\mathrm{L}$ contacts in human neurons have not been previously investigated.

Parkinson's disease (PD) is the most common neurodegenerative movement disorder characterized by progressive loss of dopaminergic neurons in the substantia nigra pars compacta $(\mathrm{SNc})$, leading to the cardinal motor symptoms of $\mathrm{PD}^{7,8}$. Interestingly, both mitochondrial and lysosomal defects have been genetically and functionally linked to $\mathrm{PD}^{9,10}$. Indeed, the lysosomal enzyme GBA1 which encodes for $\beta$-glucocerebrosidase (GCase) and catalyzes the hydrolysis of glucosylceramide (GlcCer) to glucose and ceramide, represents the greatest genetic risk factor for PD, with GBA1 mutation carriers exhibiting more severe cognitive symptoms ${ }^{11,12}$. GBA1-PD patient-derived mutant neurons demonstrate both lower GCase protein levels and reduced GCase activity ${ }^{13-16}$. Moreover, wild-type GCase activity is decreased in both idiopathic and multiple types of familial PD patient neurons ${ }^{9,16-20}$, highlighting targeting of $G B A 1$ as a potential therapeutic approach for $\mathrm{PD}^{14,16,21}$ and a key role for GCase activity in PD pathogenesis. However, whether $\mathrm{M}-\mathrm{L}$ contact dynamics are disrupted in PD patient neurons and further modulated by GCase activity is not known.

Here we investigated the impact of $\mathrm{M}-\mathrm{L}$ contact sites in neuronal function and GBA1-linked PD pathogenesis. Using superresolution time-lapse imaging, we found that $\mathrm{M}-\mathrm{L}$ contact sites dynamically formed in the soma, axon, and dendrites of human iPSC-derived dopaminergic neurons. We further reported a role for $\mathrm{M}-\mathrm{L}$ contacts in $\mathrm{PD}$ patient-derived dopaminergic neurons, as contact untethering was disrupted in patient neurons expressing heterozygous mutant GBA1. Finally, we showed that defective M-L contact untethering was caused by decreased GCase lysosomal enzymatic activity leading to GlcCer accumulation. This resulted in decreased levels of the untethering protein TBC1D15 which normally mediates Rab7 GTP hydrolysis for contact untethering. Ultimately, these defects resulted in disrupted mitochondrial distribution and function, but could be rescued by TBC1D15 in Parkinson's patient GBA1-linked neurons. Together, our work identifies an important role for M-L contact sites in human neurons and in GBA1-linked PD pathophysiology.

\section{Results}

M-L contact sites dynamically form in human neurons. To investigate $\mathrm{M}-\mathrm{L}$ contact sites in human neurons, we utilized induced pluripotent stem cell (iPSC) technology (Supplementary Fig. 1a) to generate human midbrain dopaminergic neurons from healthy controls using previously established protocols ${ }^{16,22}$. Differentiated neurons expressed the neural specific marker $\beta$-IIItubulin (TUJ1) and midbrain dopaminergic neuron-specific markers: tyrosine hydroxylase (TH), forkhead box protein A2 (FOXA2), and LIM Homeobox Transcription Factor 1 Alpha (LMX1A) (Supplementary Fig. 2a, b), and were subsequently imaged for mitochondrial and lysosomal dynamics in live neurons (Supplementary Fig. 3a, b).

Using 3D super-resolution structured illumination microscopy (3D N-SIM), we found that mitochondria and lysosomes (Mito-
RFP, Lyso-GFP) formed stable inter-organelle contacts in human neurons (Fig. 1a). Neuronal M-L contacts were further confirmed to be $<10 \mathrm{~nm}$ apart using electron microscopy (EM) (Fig. 1b), consistent with other contact sites ${ }^{2,23}$. Next, we conducted confocal time-lapse microscopy of live neurons, and found that M-L contacts dynamically formed over time and remained tethered together (yellow arrows) before subsequently untethering from one another (white arrows) (Fig. 1c and Supplemental Movies 1-3). Mitochondria in contact with lysosomes maintained their membrane potential, as visualized by TMRM imaging (Supplementary Fig. 3c-e), and dynamic M-L contact tethering could be further observed in human neurons by imaging outer mitochondrial membrane and lysosomal membrane proteins (TOM20-RFP, LAMP1-GFP) (Supplementary Fig. 3f), as well as by imaging mitochondrial and lysosomal-targeted dyes (MitoTracker, LysoTracker) (Supplementary Fig. 3g). In addition, we could also visualize $\mathrm{M}-\mathrm{L}$ contacts in human neurons by proximity ligation assay (PLA) imaging of TOM20 and LAMP1 on the outer mitochondrial and lysosomal membrane (Supplementary Fig. 4a, b). M-L contacts in human neurons remained tethered for an average duration of $88.07 \pm 5.05 \mathrm{~s}$ (Fig. 1d, e), with $\sim 18 \%$ of lysosomes in contact with mitochondria at any point in time (Fig. 1f). Thus, M-L contacts can dynamically form to mediate crosstalk between mitochondria and lysosomes in human neurons.

Spatial compartmentalization of neuronal M-L contact dynamics. Next, we investigated the spatial compartmentalization of neuronal M-L contacts in the soma, dendrites, and axons of human neurons, as both organelles are localized throughout multiple neuronal compartments. Dendrites and axons were identified as being positive for MAP2 or Tau, respectively (Supplementary Fig. 2c). Importantly, we found that mitochondria and lysosomes tethered at contact sites (yellow arrows) within all three neuronal compartments in the soma (Fig. 2a (left) and Supplemental Movie 4), dendrites (Fig. 2a (middle), Supplemental Movie 5), and axons (Fig. 2a (right) and Supplemental Movie 6). M-L contacts in dendrites and axons exhibited decreased mitochondrial and lysosomal motility (yellow arrows) (Fig. 2b). To further examine whether the dynamics of contacts differed by spatial compartmentalization, we quantified the duration of M-L contacts and percentage of lysosomes in contact with mitochondria across different neuronal compartments. Interestingly, live-cell imaging analysis revealed that the average minimum duration of $\mathrm{M}-\mathrm{L}$ contacts was significantly increased in axons compared to those in the soma (soma: $80.6 \pm 6.2 \mathrm{~s}$; dendrites: $82.4 \pm 9.7 \mathrm{~s}$; axons: $112.5 \pm 13.2 \mathrm{~s}$ ) (Fig. $2 \mathrm{c}$ ). In contrast, the percentage of lysosomes contacting mitochondria in the soma, dendrites, and axons did not differ (Fig. 2d). Together, these results demonstrate that $\mathrm{M}-\mathrm{L}$ contacts are able to form with varying dynamics across multiple neuronal compartments in human neurons.

Loss of GCase activity disrupts M-L contact untethering in GBA1-PD patient dopaminergic neurons. PD has been genetically and functionally linked to both mitochondrial and lysosomal defects $^{9,10}$, but whether M-L contacts are disrupted in PD has not been previously investigated. As mutations in the lysosomal enzyme GCase (GBA1) represent the greatest genetic risk factor for $\mathrm{PD}^{12}$, and GCase activity is decreased in both idiopathic and multiple types of familial PD patient neurons ${ }^{9,16-18}$, we examined M-L contact dynamics in GBA1-PD ( $\triangle$ het $84 \mathrm{GG}$ ) patient neurons.

Using PD patient fibroblasts harboring mutant GBA1, we generated human iPSCs and their isogenic controls by CRISPR- 
a

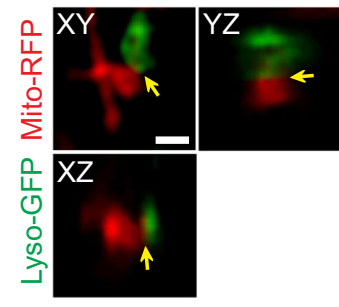

3D N-SIM

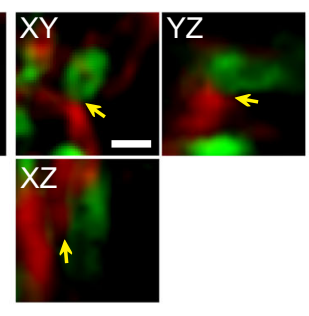

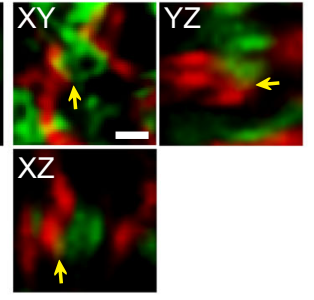

b

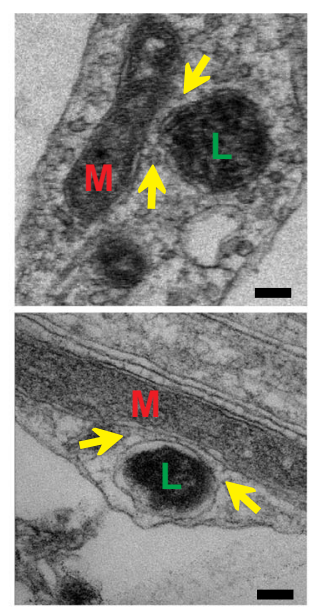

C
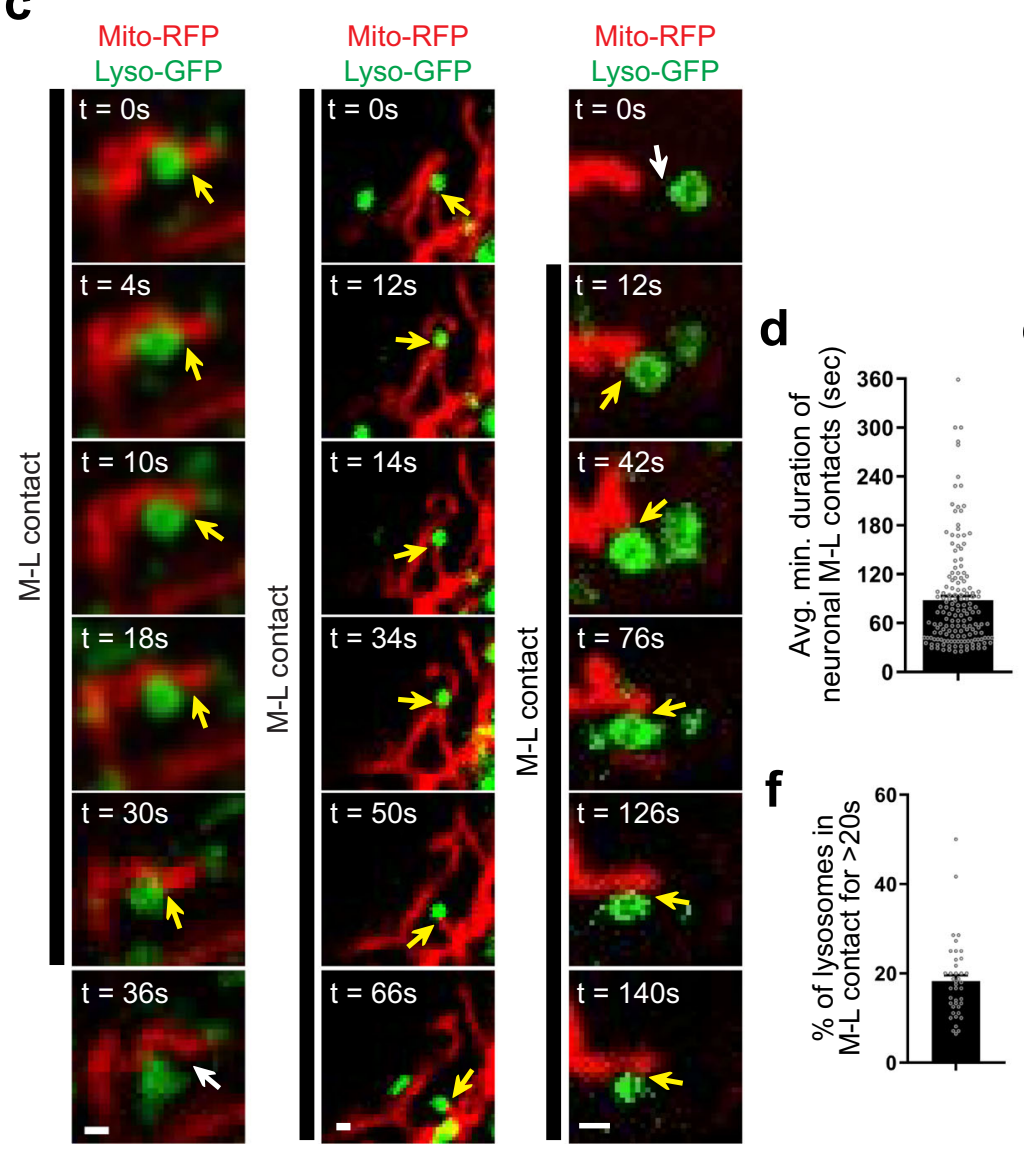

e

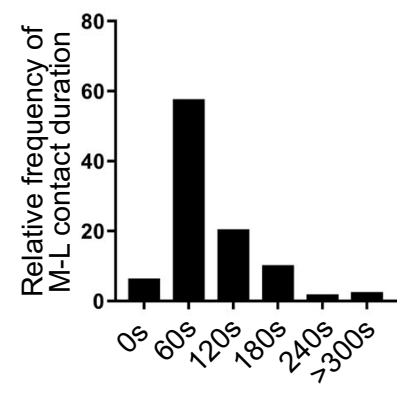

$\mathbf{f}$

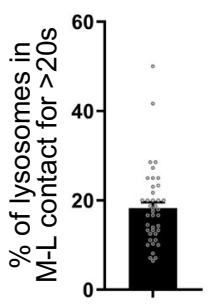

Fig. 1 Mitochondria-lysosome contacts dynamically form in human neurons. a Representative 3D structured illumination microscopy ( $N$-SIM) images of M-L contacts (yellow arrows) in wild-type human dopaminergic iPSC-derived neurons (mitochondria: red, Mito-RFP; lysosomes: green, Lyso-GFP) (30 neurons from $N=3$ independent experiments were imaged). b Representative electron microscopy (EM) images of $M-L$ contacts (yellow arrows) with distance between membranes $<10 \mathrm{~nm}$ (mitochondria, M; lysosomes, L). c Representative time-lapse confocal images of dynamic contacts between mitochondria (red, Mito-RFP) and lysosomes (green, Lyso-GFP). Time-lapse recordings were taken at $2 \mathrm{~s}$ intervals for 3-5 min. Yellow arrows mark stable $M-L$ contacts. White arrows mark the site of $M-L$ contacts before contact formation or after contact untethering. Black line shows duration of contacts (see Supplementary Movies 1 and 2). d, e Quantification of duration of stable M-L contacts from confocal images ( $n=156$ contacts from 26 neurons, $N=3$ independent experiments). d Average minimum duration of neuronal $\mathrm{M}-\mathrm{L}$ contacts. e Relative frequency (percentages) distribution of neuronal $\mathrm{M}-\mathrm{L}$ contacts. $X$-axis represents bin centers. Bin width $=60 \mathrm{~s}$. f Percentage of lysosomes contacting mitochondria (for $>20 \mathrm{~s})(n=41$ neurons, $N=3$ independent experiments). For all quantifications, data are means \pm S.E.M. Scale bars, $500 \mathrm{~nm}(\mathbf{a}, \mathbf{c}) ; 100 \mathrm{~nm}(\mathbf{b})$.

Cas916 (Supplementary Fig. 1a) which were subsequently differentiated into midbrain dopaminergic neurons (mutant GBA1 ( $\triangle \mathrm{GBA})$; isogenic control (Corr)) (Supplementary Fig. 2a-c). Both mutant GBA1 and its isogenic control did not affect the efficiencies of fibroblast reprogramming to iPSCs or differentiation into midbrain dopaminergic neurons from iPSCs ${ }^{16}$.

We first confirmed that PD patient-derived mutant GBA1 dopaminergic neurons exhibited decreased GCase protein levels (Fig. 3a, b). We also conducted live-cell GCase activity assays and showed that mutant GBA1 patient neurons had reduced total GCase enzymatic activity compared to isogenic control neurons (Fig. 3c-e). To further compare lysosomal and non-lysosomal GCase activity separately, we used Bafilomycin A1 (BafA1), an inhibitor of lysosomal acidification, as an established protocol for examining lysosomal GCase activity ${ }^{24}$ and also found a significant decrease in lysosomal GCase activity in patientderived mutant GBA1 neurons compared to CRISPR-corrected isogenic control neurons (Fig. 3c, d, f), consistent with previous findings ${ }^{16}$. 
a

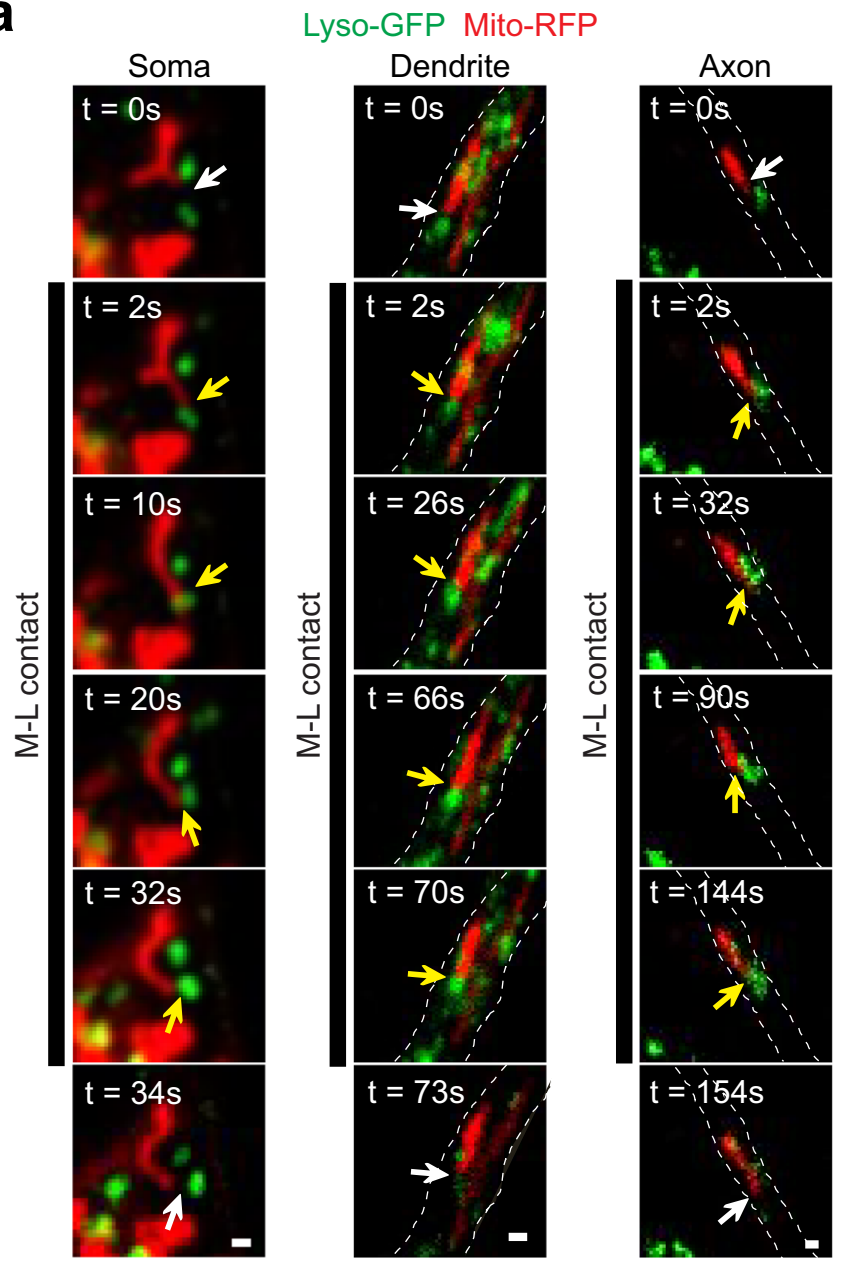

C

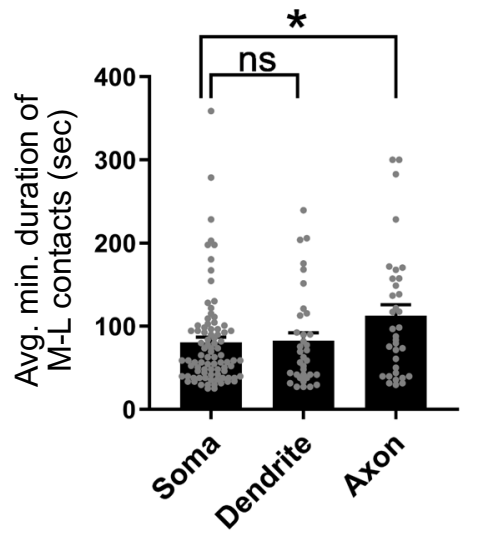

d

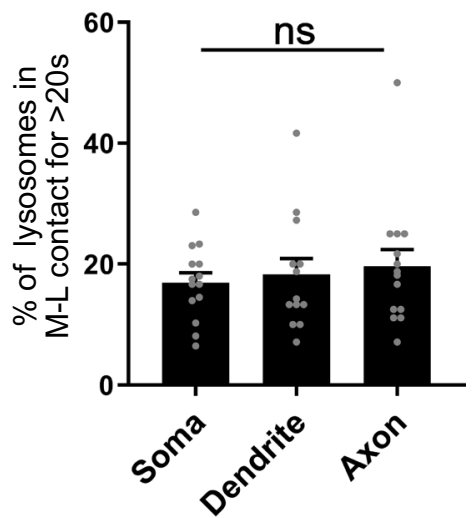

b
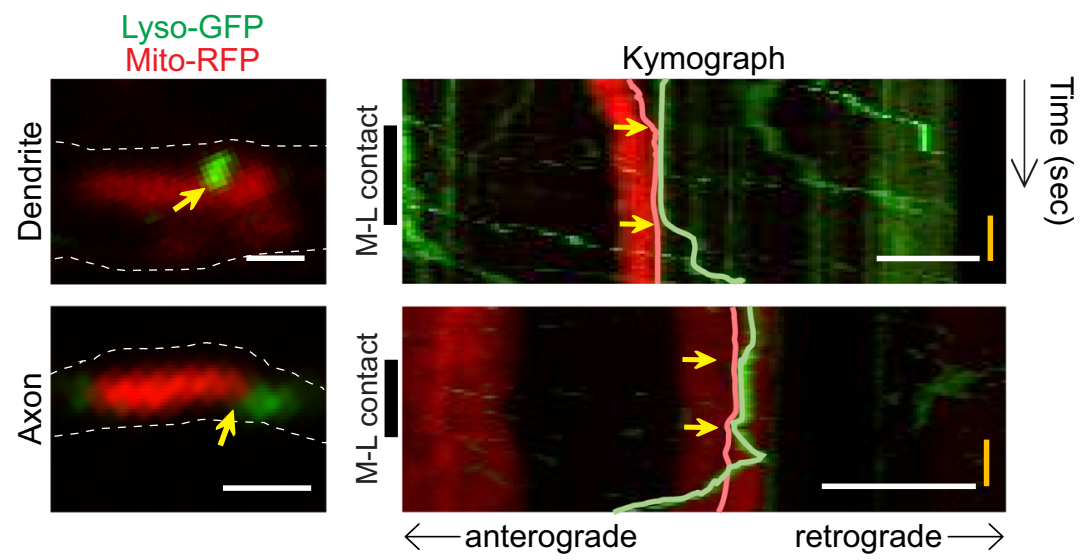

Fig. 2 Spatial compartmentalization of neuronal mitochondria-lysosome contact dynamics. a Representative time-lapse confocal images of contacts between mitochondria (red, Mito-RFP) and lysosomes (green, Lyso-GFP) in soma, dendrites, and axons of wild-type human dopaminergic iPSC-derived neurons. Time-lapse recordings were taken at $2 \mathrm{~s}$ intervals for $5 \mathrm{~min}$. Yellow arrows mark stable $\mathrm{M}-\mathrm{L}$ contacts. White arrows mark the site of $\mathrm{M}-\mathrm{L}$ contacts before contact formation or after contact untethering. Black line shows duration of contacts. Scale bar $=500$ nm (see Supplementary Movies 4-6). b Representative frames of live-cell imaging (left) and dual color kymographs (right) of M-L contacts in dendrites and axons (mitochondria: red, Mito-RFP; lysosomes: green, Lyso-GFP). In confocal image frames (left), contact sites are denoted by yellow arrows. In kymographs: white scale bar $=1 \mu \mathrm{m}$, yellow vertical bar $=30 \mathrm{~s}$. Yellow arrows in kymograph point to start and end timepoints of $\mathrm{M}-\mathrm{L}$ contact tethering. Left black line shows duration of contacts. c, d Quantification of M-L contacts across different neuronal compartments. One-way ANOVA followed by Tukey's multiple comparisons test. c Comparison of average minimum durations of M-L contacts in soma, dendrites, and axons ( $n=86$ (soma), $n=35$ (dendrite), $n=34$ (axon) contacts from 26 neurons, $N=3$ independent experiments), ${ }^{\star} p=0.0335$. d Percentage of lysosomes contacting mitochondria in soma, dendrites, and axons (for $>20 \mathrm{~s}$ ) ( $n=14$ (soma), $n=13$ (dendrite), $n=14$ (axon) neurons; $N=3$ independent experiments). For all quantifications, data are means \pm S.E.M; ${ }^{*} p \leq$ 0.05, ns: not significant. 
a

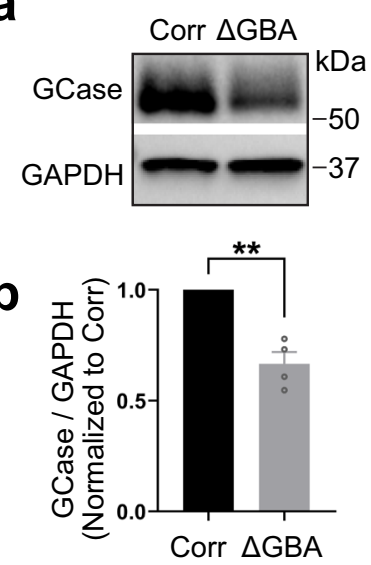

C
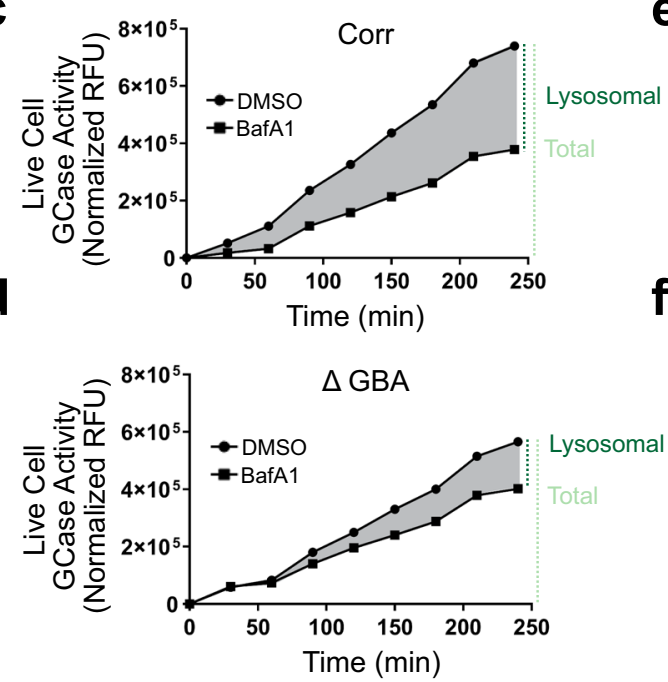

e
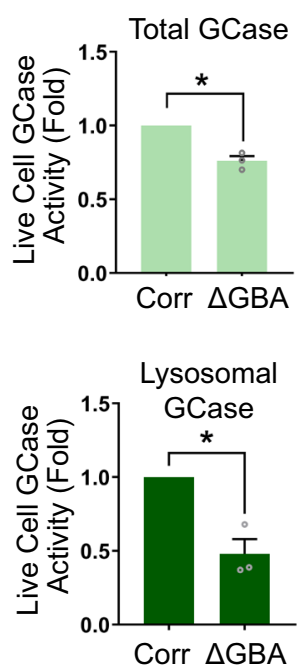

g

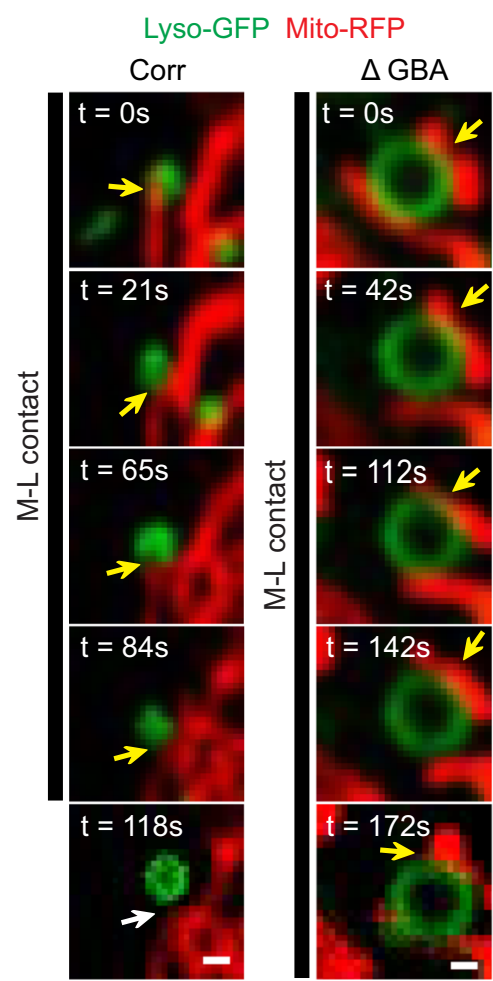

h
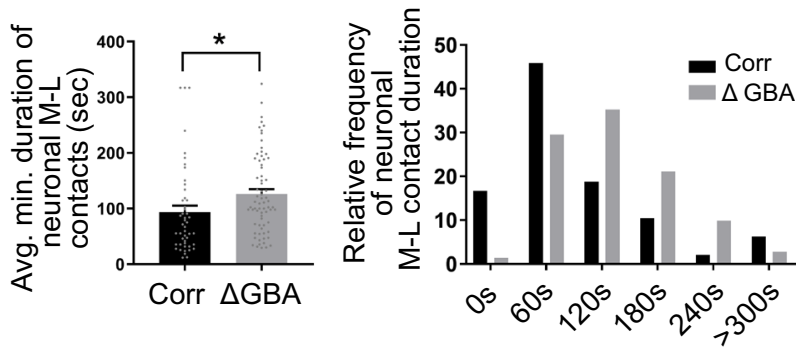

i

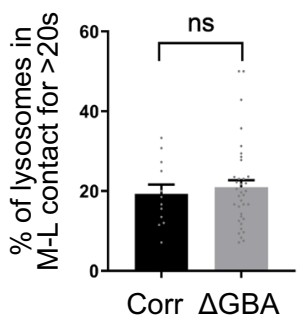

Fig. 3 Loss of GCase activity disrupts mitochondria-lysosome contact untethering in GBA1-PD patient dopaminergic neurons. a, b Western blot analysis of PD patient-derived mutant GBA1 dopaminergic neurons ( $\triangle \mathrm{GBA}$; het $84 \mathrm{GG}$ ) and its CRISPR-corrected isogenic control (Corr) neurons. GCase level was significantly reduced in $\triangle \mathrm{GBA}$ neurons $\left(N=4\right.$ independent experiments). Paired two-sided Student's $t$-test; ${ }^{\star \star} p=0.0083$. $\mathbf{c}-\mathbf{f} \Delta \mathrm{GBA}$ and Corr neurons were treated with either DMSO or BafA1 and subjected to live-cell GCase activity analysis. e Quantification of the area under each curve (AUC) demonstrates decreased total GCase activity in $\triangle$ GBA neurons. Paired two-sided Student's $t$-test; ${ }^{\star} p=0.0183$. $\mathbf{f}$ Lysosomal GCase activity was calculated by subtracting BafA1 values from DMSO. Values are expressed as fold-change compared to isogenic controls $(N=3$ independent experiments). Paired two-sided Student's $t$-test; ${ }^{*} p=0.0352$. $\mathbf{g}$ Representative time-lapse confocal images of contacts between mitochondria (red, Mito-RFP) and lysosomes (green, Lyso-GFP) in Corr (left) and $\triangle$ GBA (right) human neurons. Yellow arrows mark stable M-L contacts. White arrows mark the site of M-L contacts after contact untethering. Black line shows duration of contacts. Scale bar $=500 \mathrm{~nm}$ (see Supplementary Movies 7 and 8). $\mathbf{h}$ Quantification of average minimum duration (left) and relative frequency distribution of the duration of stable $\mathrm{M}-\mathrm{L}$ contacts (right), showing increased duration of stable $\mathrm{M}-\mathrm{L}$ contacts in $\triangle \mathrm{GBA}$ neurons ( $n=48$ contacts from Corr and $n=71$ contacts from $\triangle G B A, N=3$ independent experiments). $X$-axis of the histogram represents bin centers. Bin width $=60 \mathrm{~s}$. Unpaired two-sided Student's $t$-test; ${ }^{\star} p=0.0204$. i Percentage of lysosomes contacting mitochondria (for $>20 \mathrm{~s}$ ) ( $n=12$ Corr and $n=36 \Delta$ GBA neurons, $N=3$ independent experiments). Unpaired two-sided Student's $t$-test. For all quantifications, data are means \pm S.E. M.; ${ }^{\star} p \leq 0.05,{ }^{\star \star} p \leq 0.01$, ns: not significant.

We next investigated whether lysosomal contacts with mitochondria were disrupted by loss of lysosomal GCase activity by conducting confocal live-cell microscopy of mitochondria and lysosomes in mutant GBA1 and CRISPR-corrected isogenic control neurons. Interestingly, while M-L contacts dynamically formed in both conditions (yellow arrows) (Fig. $3 \mathrm{~g}$ and Supplemental Movies 7, 8), the average duration of M-L contact tethering was significantly increased in mutant GBA1 neurons, 
indicative of inefficient untethering events (Fig. 3h). However, the percentage of lysosomes in contacts was similar between conditions (Fig. 3i), suggesting that the subsequent untethering but not initial formation of $\mathrm{M}-\mathrm{L}$ contact tethering was disrupted in mutant GBA1 neurons.

To further examine $\mathrm{M}-\mathrm{L}$ contacts, we conducted PLA imaging of $\mathrm{M}-\mathrm{L}$ contacts in mutant GBA1 and CRISPR-corrected isogenic control neurons (Supplementary Fig. 4c), and similarly found that $\mathrm{M}-\mathrm{L}$ contacts could still form in both conditions (Supplementary Fig. 4d). In addition, we conducted EM imaging of M-L contacts in mutant GBA1 and CRISPR-corrected isogenic control neurons (Supplementary Fig. 4e), and also found that $\mathrm{M}-\mathrm{L}$ contacts tethered together in both conditions, with the length of membrane contact between mitochondria and lysosomes not altered in mutant GBA1 neurons (Supplementary Fig. 4f). Of note, ER-mitochondria contact and ER-lysosome contact formation were also not disrupted in mutant GBA1 neurons (Supplementary Fig. 5a-f). Together, our results suggest that loss of GCase activity does not disrupt M-L contact formation, but preferentially disrupts the untethering of $\mathrm{M}-\mathrm{L}$ contact sites, resulting in prolonged contact site tethering between mitochondria and lysosomes.

We further examined whether these changes in $\mathrm{M}-\mathrm{L}$ contact dynamics might be specific to loss of GCase activity rather than general lysosomal enzyme dysfunction. Mutant GBA1 neurons had altered lysosomal morphology, including enlarged lysosomes (Fig. 3g and Supplementary Fig. 3h, i). However, inhibition of other lysosomal enzymes in human neurons which also led to enlarged lysosomal morphology (Supplementary Fig. 6a) including inhibition of lysosomal acid ceramidase (carmofur treatment), cysteine proteases (E64D treatment), or aspartyl proteases (pepstatin A treatment) did not disrupt $\mathrm{M}-\mathrm{L}$ contact dynamics (Supplementary Fig. 6b). Thus, our results suggest that this pathway is selectively disrupted by loss of GCase activity rather than general lysosomal defects or enzyme dysfunction.

In addition, we assessed whether rescuing GCase activity in mutant GBA1 neurons was sufficient to restore defective $\mathrm{M}-\mathrm{L}$ contact dynamics. The modulator S-181 was recently found to increase GCase activity in mutant GBA1 neurons ${ }^{16}$. Importantly, S-181 treatment (Supplementary Fig. 6c) rescued the prolonged $\mathrm{M}-\mathrm{L}$ contact tethering in mutant GBA1 neurons (Supplementary Fig. 6d), further highlighting the role of GCase activity in regulating $\mathrm{M}-\mathrm{L}$ contact dynamics.

Tethering proteins mediating $\mathrm{M}-\mathrm{L}$ contact are disrupted in GBA1-PD patient dopaminergic neurons. We subsequently examined the potential mechanism through which mutant GBA1 might disrupt $\mathrm{M}-\mathrm{L}$ contact dynamics. We recently showed that $\mathrm{M}-\mathrm{L}$ contact untethering is mechanistically regulated by lysosomal RAB7-GTP hydrolysis from GTP to GDP-bound Rab72, and is driven by the GAP (GTPase-activating protein) activity of mitochondrial TBC1D15 (Rab7 GAP) ${ }^{3,5}$, which is recruited to the outer mitochondrial membrane by Fis $1^{4,6}$. Thus, we investigated whether RAB7-GTP hydrolysis might be disrupted by mutant GBA1 in patient neurons. We first measured the total protein levels of RAB7 together with Fis1 and TBC1D15 in PD patientderived mutant GBA1 dopaminergic neurons compared to isogenic controls (Fig. 4a-d). We observed no differences in total Rab7 levels (Fig. 4c) or Fis1 levels (Fig. 4d), and Fis1 levels were not altered even after normalizing for mitochondrial levels (Fis1/ Tom20: Corr vs GBA1, $p=0.23$ (not significant)) (Supplementary Fig. 3j). Of note, TBC1D15 and Fis1 localization to mitochondria (Supplementary Fig. 7a, b), and Rab7 localization to lysosomes (Supplementary Fig. 7c), as well as the levels of the Rab7 GEF (guanine nucleotide exchange factor) complex proteins
Mon1 and Ccz1 (Supplementary Fig. 7d) were also not altered in mutant GBA1 neurons. However, we surprisingly found that TBC1C15 levels were significantly decreased in mutant $G B A 1$ patient-derived neurons $\left({ }^{*} p \leq 0.05\right)$ (Fig. $4 \mathrm{~b}$ ).

Based on these findings, we hypothesized that disrupted $\mathrm{M}-\mathrm{L}$ contact untethering in mutant GBA1 neurons might be due to defective Rab7-GTP hydrolysis as a consequence of reduced TBC1D15 levels (Rab7 GAP). To test this, we performed GSTRILP (Glutathione Transferase-Rab Interacting Lysosomal Protein) pull-down assays ${ }^{25}$ to measure GTP-bound Rab7 levels, as RILP preferentially binds to GTP-bound Rab726,27 (Fig. 4e). Importantly, we found that PD patient-derived mutant $G B A 1$ dopaminergic neurons demonstrated significantly increased levels of RAB7-GTP/total Rab7 compared to CRISPR-corrected isogenic control neurons $\left({ }^{*} p \leq 0.05\right)$ (Fig. 4e, f). Together, these results suggest that decreased TBC1D15 levels in mutant GBA1 neurons disrupt Rab7 GTP hydrolysis, resulting in increased GTP-bound Rab7 and prolonged $\mathrm{M}-\mathrm{L}$ contact tethering dynamics.

To further investigate the downregulation of TBC1D15 in mutant GBA1 neurons, we analyzed TBC1D15 by qPCR and showed that decreased TBC1D15 protein levels in mutant GBA1 neurons were not due to lower TBC1D15 transcripts levels (Supplementary Fig. 8a). Rather, decreased TBC1D15 protein levels resulted from elevated proteasomal degradation of TBC1D15, which could be inhibited by lactacystin, leading to similar TBC1D15 protein levels between mutant GBA1 and isogenic control neurons (Supplementary Fig. 8b). We next examined whether mitochondrial dysfunction was sufficient to disrupt TBC1D15 expression levels. Treatment of either the mitochondrial uncoupler carbonyl cyanide p-trifluoromethoxyphenylhydrazone (CCCP), Complex I inhibitor rotenone, Complex III inhibitor Antimycin, or the ATP synthase inhibitor Oligomycin (Supplementary Fig. 8c-f) did not disrupt TBC1D15 expression levels in HeLa cells. In contrast, increasing GCase activity in mutant GBA1 neurons using the modulator S$181^{16}$ was sufficient to rescue the decreased levels of TBC1D15 (Supplementary Fig. 6e, f). Thus, these findings suggest that TBC1D15 levels are downregulated at the protein level by proteosomal degradation in mutant GBA1 neurons, due to the loss of GCase activity rather than general mitochondrial dysfunction.

Finally, we examined if loss of TBC1D15 was able to disrupt $\mathrm{M}-\mathrm{L}$ contact dynamics in human neurons. Knockdown of TBC1D15 in wild-type iPSC-derived neurons (Supplementary Fig. 9a) increased M-L contact tethering duration (Supplementary Fig. 9b), consistent with findings in non-neuronal cells ${ }^{2}$ and further supporting our results that decreased TBC1D15 levels in PD GBA1-linked patient neurons disrupt $\mathrm{M}-\mathrm{L}$ contact dynamics.

Inhibition of GCase activity disrupts M-L contact untethering. To further examine whether M-L contact untethering defects were indeed dependent on loss of GCase activity, we treated wildtype human midbrain dopaminergic neurons expressing wildtype GCase with the GCase inhibitor conduritol-b-epoxide (CBE) $(50 \mu \mathrm{M} ; 7$ days) which decreases neuronal GCase activity and promotes GlcCer accumulation ${ }^{14,16,28}$. Using confocal live-cell time-lapse imaging of mitochondria and lysosomes in both wildtype (Ctrl) and CBE-treated (+CBE) human neurons, we found that M-L contacts dynamically formed in both conditions (yellow arrows) (Fig. 5a). In addition, CBE treatment was effective in increasing lysosomal size as has been previously reported ${ }^{29}$ (Fig. 5b, c). Importantly, we found that inhibition of GCase activity by CBE treatment resulted in significantly prolonged $\mathrm{M}-\mathrm{L}$ contact tethering duration $\left({ }^{* * *} p \leq 0.001\right)$ (Fig. $\left.5 \mathrm{a}, \mathrm{d}\right)$, consistent with what we observed in mutant GBA1 patient-derived neurons, and further confirming the effect of decreased GCase activity on disrupting $\mathrm{M}-\mathrm{L}$ contact untethering. 
a

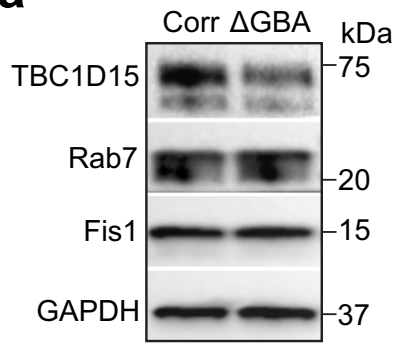

e

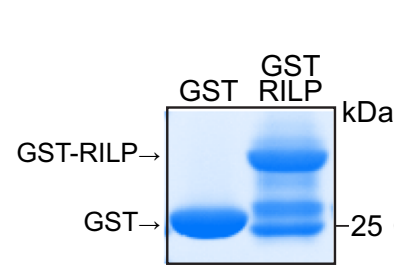

b

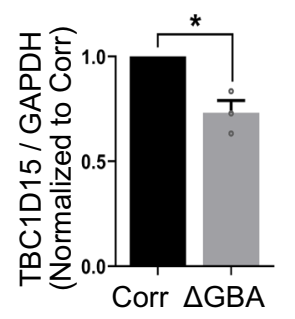

C

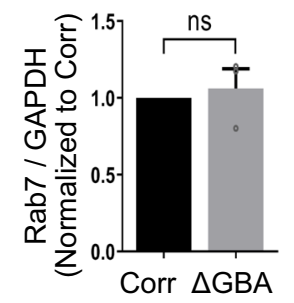

d

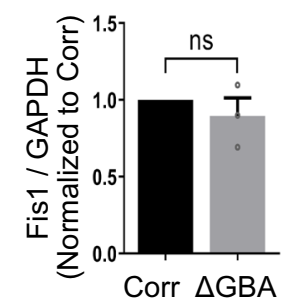


a

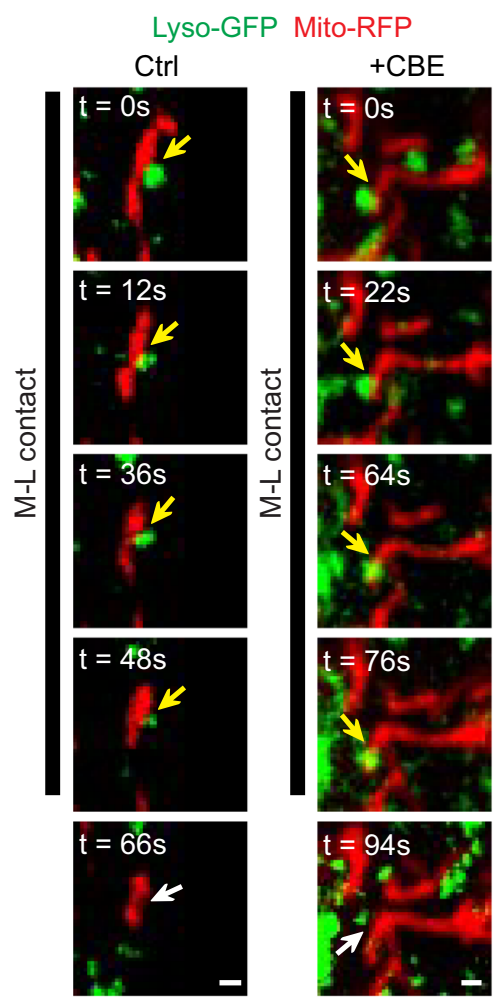

b

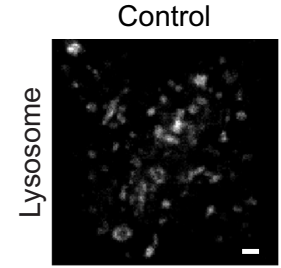

C

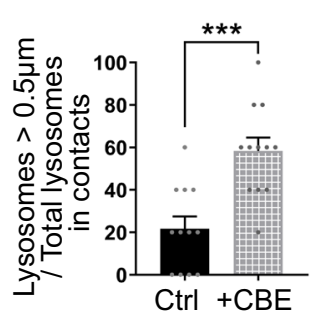

$+\mathrm{CBE}$

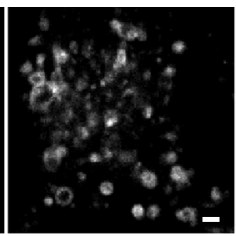

d

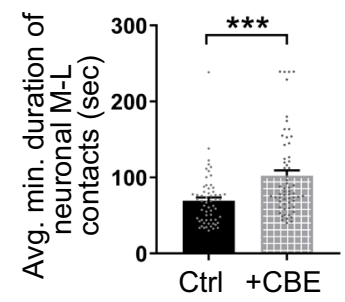

$+\mathrm{CBE}$

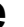

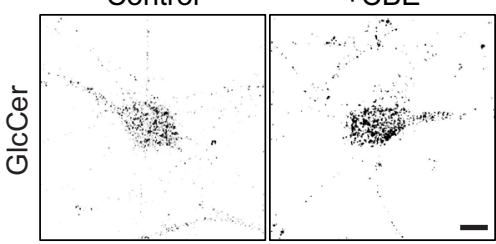

f

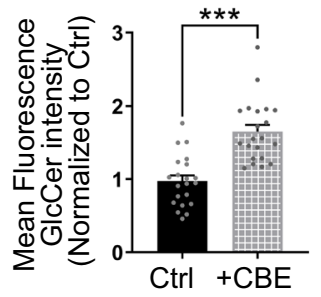

9

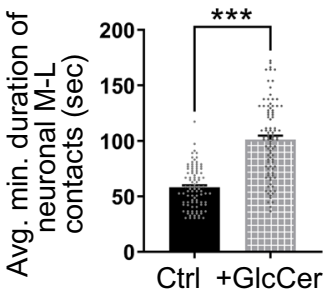

h
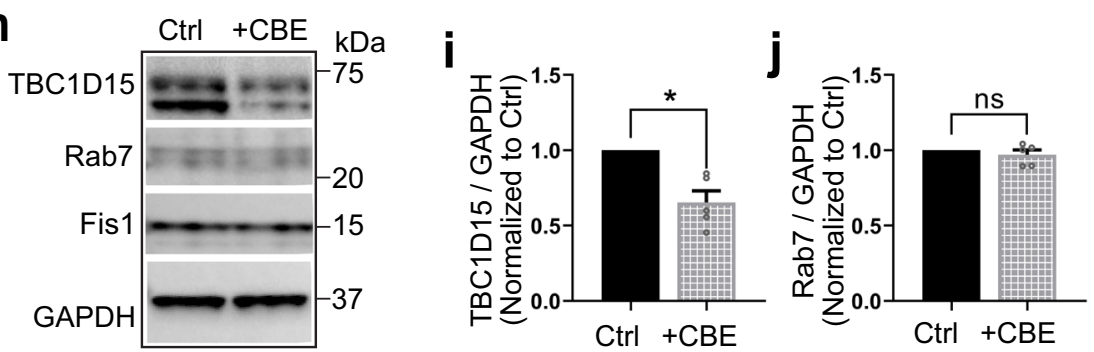

k

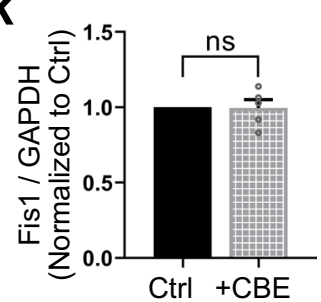

|

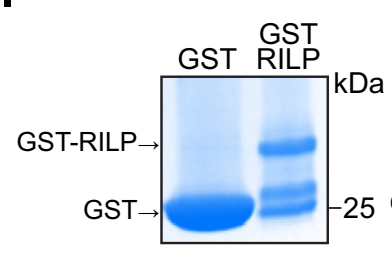

Pull-down output
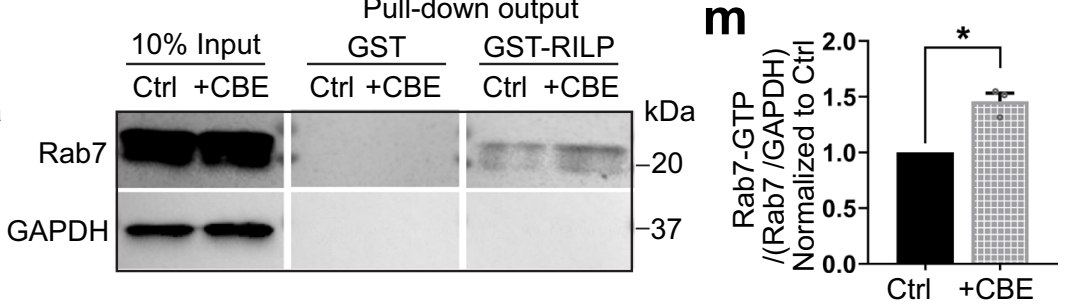

might contribute to these defects in mitochondrial distribution and function. Thus, to test if these defects could be rescued by promoting $\mathrm{M}-\mathrm{L}$ contact untethering in mutant GBA1 neurons, we expressed human TBC1D15 in mutant GBA1 neurons by lentiviral transduction (Fig. $6 \mathrm{~g}$ and Supplementary Fig. 11a). The expression of exogenous TBC1D15 in neurons was confirmed by western blot analysis and fluorescence imaging (Supplementary Fig. 11b, c). Importantly, consistent with our previous findings that TBC1D15 promotes $\mathrm{M}-\mathrm{L}$ contact untethering ${ }^{2}$, transduction of wild-type TBC1D15 in mutant GBA1 neurons ( $\triangle \mathrm{GBA}$ $+\mathrm{TBC} 1 \mathrm{D} 15)$ was able to promote $\mathrm{M}-\mathrm{L}$ contact untethering, resulting in significantly decreased $\mathrm{M}-\mathrm{L}$ contact durations compared to lentiviral vehicle-treated neurons $(\triangle \mathrm{GBA}+\mathrm{veh})$ (***p $\leq 0.001)$ (Fig. 6h).

We further investigated the effect of rescuing $\mathrm{M}-\mathrm{L}$ contact dynamics on axonal mitochondrial density in mutant GBA1 neurons. Interestingly, while TBC1D15 expression did not alter mitochondrial densities in the soma of mutant GBA1 neurons (Fig. 6g, i), it significantly rescued the decreased mitochondrial density in axons of mutant $G B A 1$ neurons (Fig. 6g, j). Moreover, TBC1D15 expression was also able to partially rescue ATP levels in mutant GBA1 neurons (Fig. 6k). Together, our results suggest that upregulation of TBC1D15 in PD patient mutant GBA1 neurons is sufficient to rescue the misregulation of $\mathrm{M}-\mathrm{L}$ contact 
Fig. 5 Inhibition of GCase activity disrupts mitochondria-lysosome contact untethering in dopaminergic neurons. a Healthy control WT human iPSCderived dopaminergic neurons were treated with vehicle (CtrI) or CBE (50 $\mu \mathrm{M} ; 7$ days) to inhibit GCase activity. Representative time-lapse confocal images of contacts between mitochondria (red, Mito-RFP) and lysosomes (green, Lyso-GFP) in Ctrl (left) and CBE-treated (right) human neurons. Yellow arrows mark stable $M-L$ contacts. White arrows mark the site of $M-L$ contacts after contact untethering. Black line shows duration of contacts. Scale bar $=500$ nm. b Representative confocal images of lysosomes (LAMP1-GFP) in the Ctrl (left) and CBE-treated (right) human neurons. Scale bar $=1 \mu \mathrm{m}$. $\mathbf{c}$ The effects of $\mathrm{CBE}$ treatment on lysosomal morphology were confirmed by quantification of the percentage of lysosomes that were enlarged (diameter $>0.5 \mu \mathrm{m}$ ) in M-L contacts ( $n=12$ neurons per condition, $N=3$ independent experiments). Unpaired two-sided Student's $t$-test; ${ }^{\star \star \star} p=0.0001$. d CBE treatment in control neurons increased the average minimum duration of stable $M-L$ contacts ( $n=60$ contacts per condition, $N=3$ independent experiments). Unpaired two-sided Student's $t$-test; ${ }^{\star \star \star} p=0.0001$. e Representative confocal images of immunocytochemistry of GlcCer in the Ctrl (left) and CBE-treated (right) human neurons. Scale bar $=5 \mu \mathrm{m}$. $\mathbf{f}$ Quantification of CBE treatment leading to increased GlcCer levels as measured by immunofluorescence signal of GlcCer ( $n=21$ neurons per condition, $N=3$ independent experiments). $\mathbf{g}$ Exogenous GlcCer treatment in control neurons increased the average minimum duration of stable M-L contacts ( $n=93$ contacts from Ctrl, $n=94$ contacts from + GlcCer neurons, $N=3$ independent experiments). $\mathbf{f}$, $\mathbf{g}$ Unpaired two-sided Student's $t$-test; ${ }^{\star \star \star} p<0.0001$. h-k Western blot analysis of $\mathbf{i}$ TBC1D15, $\mathbf{j}$ Rab7, and $\mathbf{k}$ Fis1 levels in Ctrl and CBE-treated neurons. Protein levels were normalized to loading control GAPDH. Values are expressed as fold-change compared to $\mathrm{Ctrl}(\mathrm{N}=5$ independent experiments). Paired two-sided Student's $t$-test; ${ }^{\star} p=0.0104$. I, m GST-RILP pull-down to measure GTP-bound Rab7 levels in Ctrl and CBE-treated neurons. m Rab7-GTP levels were normalized to total Rab7 normalized to GAPDH. Values are expressed as fold-change compared to $\mathrm{Ctrl}$ ( $N=3$ independent experiments). Paired twosided Student's $t$-test; ${ }^{\star} p=0.0242$. For all quantifications, data are means \pm S.E.M., ${ }^{\star} p \leq 0.05,{ }^{\star \star \star} p \leq 0.001$.

untethering, as well as downstream defects in mitochondrial dynamics and function.

Finally, we extended our analysis to additional PD mutant GBA1 patient neurons ( $\triangle$ het N370S) and CRISPR-edited isogenic control neurons (Supplementary Fig. 12a-c). Mutant GBA1 (N370S) patient neurons also showed decreased GCase levels and enzymatic activity (Supplementary Fig. 12d-g). Importantly, they also had significantly increased GTP-bound Rab7 due to lower TBC1D15 levels (Supplementary Fig. 12h-m), which resulted in prolonged $\mathrm{M}-\mathrm{L}$ contact tethering (Supplementary Fig. 12n). Moreover, S-181 modulator treatment in mutant GBA1 (N370S) neurons was sufficient to rescue both M-L contact dynamics (Supplementary Fig. 12n) as well as TBC1D15 levels (Supplementary Fig. 120). In addition, mutant GBA1 (N370S) neurons also demonstrated reduced mitochondrial function (Supplementary Fig. 12p). Thus, these findings further highlight the role of defective GCase activity in PD GBA1-linked patient neurons on disrupting Rab7 GTP hydrolysis machinery and M-L contact site dynamics.

\section{Discussion}

Our study demonstrates that $\mathrm{M}-\mathrm{L}$ contact sites dynamically form in human neurons, and further investigates their role in neurons from patients with GBA1-linked PD. We found that loss of lysosomal GCase enzymatic activity in PD patient-derived dopaminergic neurons led to prolonged $\mathrm{M}-\mathrm{L}$ contact tethering dynamics due to defective contact untethering machinery, and resulted in misregulated axonal distribution of mitochondria and decreased ATP levels. Importantly, we showed that rescuing M-L contact site dynamics in PD patient neurons is sufficient to ameliorate defects in mitochondrial distribution and function, thus highlighting a potential role for $\mathrm{M}-\mathrm{L}$ contact site dysregulation in PD pathogenesis.

Multiple genes linked to mitochondria or lysosomes have been identified as causative or risk genes of $\mathrm{PD}^{33,34}$. Moreover, both mitochondrial and lysosomal dysfunction have been implicated in

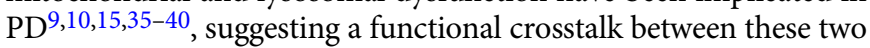
organelles. We previously found that oxidized dopamine mediates convergence of mitochondrial and lysosomal dysfunction in human but not in mouse dopaminergic neurons ${ }^{9}$, highlighting the importance of patient-derived dopaminergic neurons for studies of PD pathogenesis.

Despite the previously studied role of mitochondria and lysosomes in PD pathogenesis, a direct homeostatic relationship between these two organelles that is independent of eventual lysosomal degradation of mitochondria has not been examined. In this context, our work provides evidence for the role of $\mathrm{M}-\mathrm{L}$ contacts not only in the homeostasis of dopaminergic neurons but also as a link between mitochondrial and lysosomal dysfunction in PD pathogenesis. We specifically focused on $G B A 1$ mutations which represent the greatest genetic risk factor for $\mathrm{PD}^{11,12}$. Importantly, wild-type GCase enzyme activity is also reduced in patient neurons with genetic or idiopathic PD who do not harbor GBA1 mutations $9,16,18$, suggesting that loss of GCase activity is an important contributor to PD pathogenesis. Previously, we reported that the loss of GCase function in patient neurons compromises lysosomal protein degradation ${ }^{15}$ which contributes to other key PD pathogenic phenotypes including $\alpha$ synuclein accumulation $13,15,24,41$. In addition to its primary lysosomal dysfunction, GBA1 mutations and abnormal GCase activity have also been linked to mitochondrial dysfunction $9,28,42-46$.

In this study, we show that GBA1 mutant PD patient neurons have defective $\mathrm{M}-\mathrm{L}$ contact dynamics, resulting in prolonged contact tethering. While previous studies did not examine the GTP-bound state of Rab7 $7^{47,48}$, we found that loss of GCase activity resulted in an increased percentage of active GTP-bound lysosomal Rab7, which directly mediates M-L contact tethering2 This is likely due to decreased levels of TBC1D15 (Rab7 GAP) in mutant GBA1 patient neurons, as TBC1D15's GAP activity promotes Rab7 GTP hydrolysis and subsequent M-L contact untethering ${ }^{2}$. Indeed, we found that increasing TBC1D15 expression was sufficient to rescue the prolonged M-L contact tethering we observed in mutant GBA1 neurons. In addition, we further observed mitochondrial dysfunction in patient neurons, including abnormal mitochondrial distribution in axons, decreased mitochondrial respiration and lower ATP levels. Of note, TBC1D15 expression was also able to rescue mitochondrial dysfunction. Thus, dysregulation of $\mathrm{M}-\mathrm{L}$ contacts may play an important role in GBA1-linked PD pathogenesis, and targeting contact machinery may help ameliorate downstream mitochondrial dysfunction. Given that mitochondria play key roles as energy suppliers especially in the synapses of active neurons, we hypothesize that such abnormal distribution of mitochondria may further contribute to synaptic dysfunction in $\mathrm{PD}^{10}$.

While inter-organelle contact sites have been found to be essential subdomains for modulating cellular function and homeostasis ${ }^{49-51}$, only recently have studies reported the function and molecular architecture of inter-organelle contacts in neurons, such as those between the mitochondria and endoplasmic reticulum ${ }^{40,52,53}$. In addition, dysfunction of inter-organelle 
a

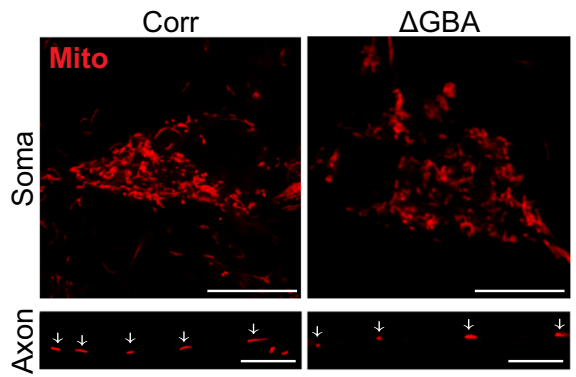

b

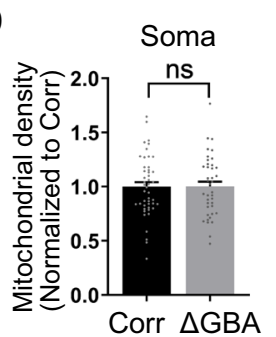

$\mathbf{C}$

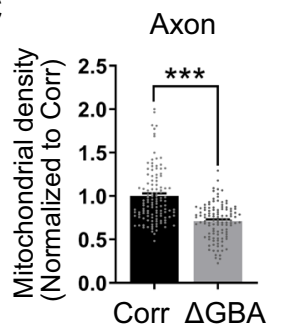

d

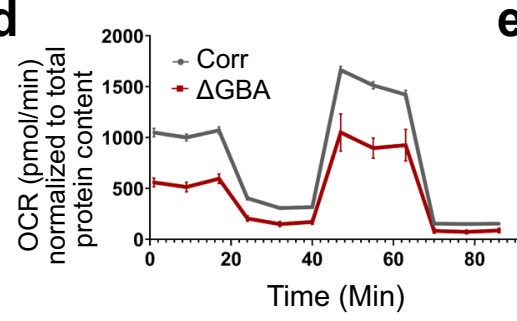

e

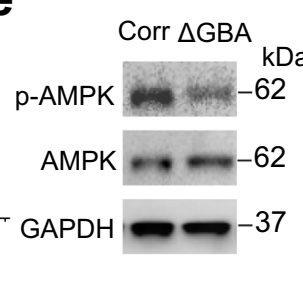

f

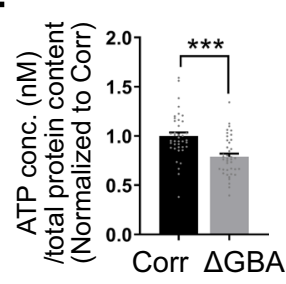

g

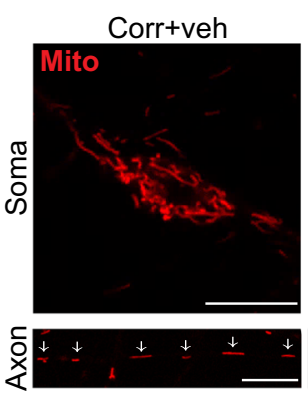

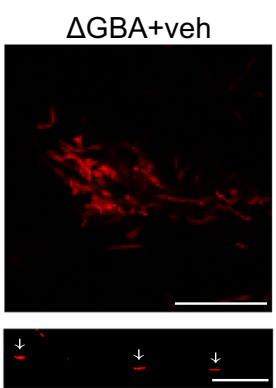

h

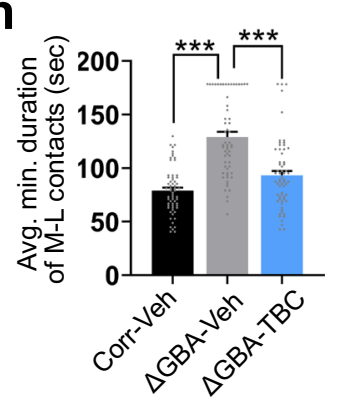

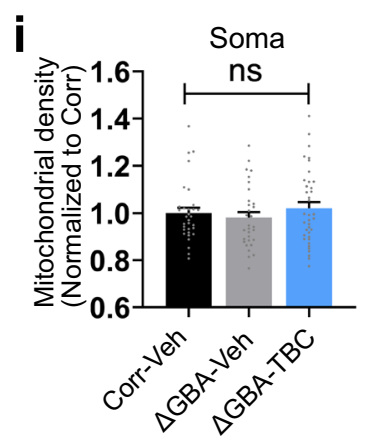
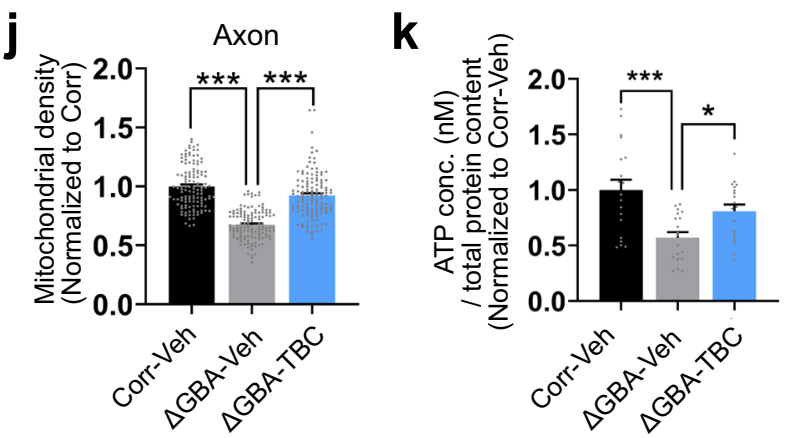

Fig. 6 Mitochondrial dysfunction due to prolonged mitochondria-lysosome contacts is partially rescued by expression of TBC1D15 in GBA1-PD patient dopaminergic neurons. a Live-cell distribution of mitochondria (TMRM, red) in the soma and axons from PD patient-derived mutant GBA1 dopaminergic neurons ( $\triangle \mathrm{GBA}$ ) and its CRISPR-corrected isogenic control (Corr) neurons. White arrows mark mitochondria in a single axon. Scale bar, $10 \mu \mathrm{m}$. $\mathbf{b}$ Quantified mitochondrial density in the soma (TMRM-positive pixels/pixels of soma). Corr ( $n=47$ neurons), $\Delta$ GBA $(n=47$ neurons) ( $N=3$ independent experiments). c Quantified mitochondrial density in axons (mitochondria count/length of axon $(\mu \mathrm{m}))$. Corr $(n=122$ axons), $\Delta \mathrm{GBA}(n=114$ axons) ( $N=3$ independent experiments). b, c Unpaired two-sided Student's $t$-test; ${ }^{\star \star \star} p<0.0001$. d Oxygen consumption rate (OCR) was measured and normalized to total protein content. Corr ( $n=9$ samples), $\triangle$ GBA ( $n=9$ samples) $(N=3$ independent experiments). e Western blot analysis of phospho-AMPK $\alpha$ and AMPK $\alpha$ levels in Corr and $\triangle$ GBA neurons. Ratio of $\mathrm{p}$-AMPK $\alpha / A M P K \alpha$ are expressed as fold-change compared to Corr ( $N=3$ independent experiments). Paired two-sided Student's $t$-test; ${ }^{\star} p=0.0354$. $\mathbf{f}$ Total cellular ATP content was measured and normalized to total protein content $(\mathrm{ng} / \mu \mathrm{l})$. Corr $(n=$ 40 samples), $\triangle \mathrm{GBA}$ ( $n=40$ samples) ( $N=3$ independent experiments). Unpaired two-sided Student's $t$-test; ${ }^{* \star *} p<0.0001$. $\mathbf{g}$ Live-cell distribution of mitochondria (TMRM, red) in the soma and axons from Corr and $\triangle G B A$ human neurons with or without human TBC1D15 lentiviral expression. White arrows mark mitochondria in a single axon. Scale bar, $10 \mu \mathrm{m}$. h Quantification of average minimum duration of $\mathrm{M}-\mathrm{L}$ contacts in Corr-vehicle $(n=62$ contacts from 18 neurons), $\Delta$ GBA-vehicle ( $n=60$ contacts from 15 neurons), $\triangle$ GBA-TBC1D15 ( $n=63$ contacts from 19 neurons) $\left(N=3\right.$ independent experiments); ${ }^{\star \star \star} p<$ 0.0001. i Quantified mitochondrial density in the soma (TMRM-positive pixels/pixels of soma). Corr-vehicle $(n=32$ neurons), $\Delta$ GBA-vehicle $(n=31$ neurons), $\triangle$ GBA-TBC1D15 ( $n=38$ neurons) ( $N=3$ independent experiments). j Quantified mitochondrial density in axons (mitochondria count/length of axon $(\mu \mathrm{m})$ ). Corr-vehicle $\left(n=124\right.$ axons), $\Delta$ GBA-vehicle $\left(n=127\right.$ axons), $\Delta$ GBA-TBC1D15 $\left(n=127\right.$ axons) $\left(N=3\right.$ independent experiments), ${ }^{\star \star \star} p<0.0001$. k Total cellular ATP concentration was measured and normalized to total protein content $(n g / \mu)$ ). Corr-vehicle $(n=19$ samples), $\Delta$ GBA-vehicle $(n=$ 19 samples), $\triangle$ GBA-TBC1D15 ( $n=19$ samples) ( $N=3$ independent experiments), ${ }^{\star \star *} p=0.0002,{ }^{\star} p=0.0493$. h-k One-way ANOVA followed by Tukey's multiple comparisons test. For all quantifications, data are the means \pm S.E.M.; ${ }^{\star} p \leq 0.05,{ }^{\star \star \star} p \leq 0.001$, ns: not significant. 
contacts in disease have been shown to be key contributors in the development of various diseases $1,30,31,40,54-60$. Moreover, the recent identification of $\mathrm{M}-\mathrm{L}$ contact sites has shed new light on the direct relationship between mitochondria and lysosomes in a pathway independent of lysosomal degradation of mitochondria ${ }^{2,31,61-64}$, allowing for direct crosstalk and regulation of both organelles in a dynamic manner ${ }^{1}$. In our previous study, we showed the formation of M-L contacts in non-neuronal cells and identified protein mediators responsible for contact untethering ${ }^{1,2}$. Here, we demonstrate that $\mathrm{M}-\mathrm{L}$ contacts are also key contributors in human neurons and that contacts dynamically form in multiple neuronal compartments, suggesting that they act as important sites for the neuronal regulation of mitochondrial and lysosomal dynamics. Together, our findings not only provide insights into inter-organelle contacts in maintaining the cellular homeostasis of human neurons, but also suggest the importance of $\mathrm{M}-\mathrm{L}$ contacts as a potential target for therapeutic development in PD.

\section{Methods}

Human iPSC culture and characterization. Detailed procedures for iPSC culture and neuronal differentiation have been described previously ${ }^{16}$. Healthy control and PD patient skin fibroblasts ${ }^{16}$ (GBA1 heterozygous $84 \mathrm{GG}$ mutation (c.84dupG frameshift mutation) which prevents the expression of the mutant allele, resulting in reduced GCase levels arising from a single wild-type copy of GBA1; and GBA1 heterozygous p.N370S mutation) were reprogrammed into iPSCs through Northwestern University Stem Cell Core Facility, using Sendai virus (SeV)-based delivery of four Yamanaka factors (Oct, Sox2, Klf4, and c-Myc). We also generated an isogenic control iPSC line by correcting the mutation using CRISPR/Cas9 protocols ${ }^{65}$ as recently described ${ }^{16}$. Guide RNAs targeting the mutation were cloned into vector PX461 (Addgene \#48140) carrying the cDNA encoding for GFPtagged Cas9 nuclease. The plasmid was electroporated into GBA1 84GG mutant iPSCs together with ssODN carrying the corrected sequence. After $48 \mathrm{~h}$, generation of an isogenic control line was confirmed by FACS-sorting and sequencing. All iPSCs were maintained either in $\mathrm{mTeSR}^{\mathrm{m} w} 1$ or $\mathrm{mTeSR}^{\mathrm{m}}$ Plus media (Stemcell Technologies, \#85850, \#05825) and cells were passaged as small chunks every 6-8 days depending on confluence. All iPSC lines have been routinely characterized for expression of pluripotency markers. Cells were plated on PDL-coated coverslips and subjected to immunofluorescence staining for NANOG, OCT4, SSEA4, and TRA1-81. Genomic integrity was confirmed as described previously ${ }^{16}$. Mycoplasma tests were performed on a monthly basis to maintain qualified iPSC lines.

\section{Generation and characterization of hiPSC-derived dopaminergic neurons}

Human dopaminergic iPSC-derived neurons were differentiated using previously established protocol ${ }^{22}$ for analysis of M-L contacts. Briefly, after plating single iPSCs, cells were treated with factors according to the original protocol. At day 13 of differentiation, cells were passaged en bloc (size of 1-2 mm) onto 10$\mathrm{cm}$ culture dishes pre-coated with poly-d-lysine (PDL) (Sigma, \#P1149) / laminin (Invitrogen, \#23017-015). At day 25, neurons were treated with accutase and passaged onto PDL/laminin-coated culture dishes, and subjected to dopaminergic marker characterization through immunocytochemistry (ICC) using TH, FOXA2, LMX1A together with neural specific marker $\beta$-III-tubulin (TUJ1) at day 30 . After day 50 , neurons were considered mature and maintained in Neurobasal media (Life Technologies, \# 21103049) containing Neurocult SM1 (Stemcell Technologies, \#5711). Neurons at day 50-60 were used for immunostaining, EM, live-cell time-lapse confocal imaging, live-cell or fixed-cell super-resolution SIM. Neurons from $N \geq 3$ independent experiments (biological replicates; batches of neuron differentiation) per condition were used for each experiment.

Immunocytochemistry. Cells were immunostained for multiple antibodies depending on the purpose of each experiment. Neurons were plated on PDL/ laminin-coated coverslips and fixed in 4\% paraformaldehyde in PBS for $20 \mathrm{~min}$ and permeablized with $10 \%$ FBS and $0.1 \%$ saponin in PBS at room temperature. For GlcCer staining, $10 \%$ FBS was substituted with $2 \%$ gelatin (Sigma, \#G1393). Cells were then immuno-labeled with the following primary antibodies: Oct4 (Abcam, \#19857, 1:100); SSEA1 (Millipore, MAB\#4304, 1:100); Nanog (R\&D systems, \#AF1997, 1:50); Tra-1-81 (Millipore, \#MAB4381, 1:100); TH (Calbiochem, \#657012, 1:500); $\beta$-III-tubulin (Biolegend, \#801202, \#802001, 1:1000); Lmx1a (Milipore, MAB\#10533, 1:500); FoxA2 (Santa Cruz, \#sc-101060, 1:100); Tom20 (Abcam, \#78547, 1:100); Lamp1 (Santa Cruz, \#sc-20011, 1:100); Map2 (Novus Biological, \#NB300213, 1:3000); Tau (DAKO, \#A002401-2, 1:300); GlcCer (Glycobiotech, \#RAS_0011, 1:100). After overnight incubation at $4{ }^{\circ} \mathrm{C}$, coverslips were washed three times with PBS for $5 \mathrm{~min}$ each, incubated in Alexa-conjugated secondary antibodies (Invitrogen, \#A21206, \#A21202, \#A11029, \#A11011, \#A10042, \#A10037, \#A21449, 1:1000) for $1 \mathrm{~h}$ at room temperature, washed three times and mounted onto Superfrost Plus microscope slides (Fisherbrand, \#12-55015) with VECTASHIELD HardSet Antifade Mounting Medium (Vector Labs, \#H1400). Alternatively, ProLong ${ }^{\text {tw }}$ Diamond Antifade Mountant (Thermo Fisher Scientific, \#P36959) was used for SIM sample preparation. Images were obtained on either a Leica DMI4000B confocal microscope using Leica Application Suite X (Leica) or a Nikon A1R laser scanning confocal microscope with GaAsp detectors using NIS-Elements (Nikon). Colocalization was measured using the EzColocalization plugin in ImageJ (National Institutes of Health (NIH)) ${ }^{66}$.

Proximity ligation assay. Proximity between outer mitochondrial membrane protein Tom 20 and lysosomal membrane protein Lamp1 was detected using Duolink $^{\text {tw }}$ PLA kit (Sigma Aldrich, \#DUO92101) according to the manufacturer's protocol. Neurons were plated on PDL/laminin-coated coverslips and cells were immuno-labeled with Tom20 (Abcam, \#78547, 1:100) and Lamp1 (Santa Cruz, \#sc-20011, 1:100). Images of red PLA signals were collected using Nikon A1R laser scanning confocal microscope with GaAsp detectors.

Electron microscopy. For EM, neurons were grown on PDL/laminin-coated glass coverslips. Neurons were fixed with $2.5 \%$ glutaraldehyde and $2 \%$ paraformaldehyde in $0.1 \mathrm{M}$ cacodylate buffer, $\mathrm{pH} 7.3$ for $30 \mathrm{~min}$ at room temperature, and then submitted to the Northwestern EM core facility for subsequent processing. After post-fixation in $1 \%$ osmium tetroxide and 3\% uranyl acetate in PBS, cells were dehydrated in an ethanol series, embedded in Epon resin and polymerized for $48 \mathrm{~h}$ at $60^{\circ} \mathrm{C}$. Ultrathin sections were made using a UCT ultramicrotome (Leica Microsystems) and contrasted with $4 \%$ uranyl acetate and Reynolds's lead citrate. Samples were imaged on a FEI Tecnai Spirit G2 transmission electron microscope (FEI) operated at $80 \mathrm{kV}$. Images were captured with an Eagle 4k HR 200kV CCD camera and analyzed using ImageJ (NIH).

Live-cell time-lapse confocal microscopy. Live-cell time-lapse confocal imaging was conducted of mitochondria and lysosomes labeled with the following reagents: Lentivirus expressing Tom20-RFP or Lamp1-GFP (MOI = 3, 3 days); CellLight ${ }^{\circ}$ BacMam 2.0 baculovirus (PPC (particles per cell) $=40,2$ days) [Thermo Fisher: Lysosomes-GFP (\#C10596), Lysosomes-RFP (\#C10597), Mitochondria-RFP (\# C10601), ER-GFP (\#C10590)]; LysoTracker ${ }^{\mathrm{mm}}$ Red DND-99 ( $2 \mu \mathrm{M}, 45 \mathrm{~min}$; Thermo Fisher, \#L7528); MitoTracker ${ }^{\text {mo }}$ Green FM $(0.125 \mu \mathrm{M}, 45 \mathrm{~min}$; Thermo Fisher, \#M7514); and TMRM (0.1 $\mu \mathrm{M}, 45 \mathrm{~min}$; Fisher Scientific, \#T669), which is a cellpermeant dye that accumulates in active mitochondria with intact membrane potential $(\Delta \Psi \mathrm{m})$. For analysis of lysosomal-drug-treated cultures, neurons were treated with GCase inhibitor CBE (50 $\mu \mathrm{M}, 7$ days) (Cayman Chemicals, \#15216), carmofur $(7.5 \mu \mathrm{M}, 2$ days) (Cayman Chemicals, \#14243), E-64D $(10 \mu \mathrm{M}, 24 \mathrm{~h})$ (Cayman Chemicals, \#13533), or Pepstatin A $(10 \mu \mathrm{M}, 24 \mathrm{~h}$ ) (Milipore, \#516481). For live imaging, neurons were grown on glass-bottomed culture dishes (MatTek, \#P35G-1.5-14-C) in Neurobasal media (Life Technologies, \#21103049) containing Neurocult SM1 (Stemcell Technologies, \#5711). Samples were imaged on a Nikon A1R laser scanning confocal microscope with GaAsP detectors using a Plan Apo $\lambda$ $100 \times 1.45 \mathrm{NA}$ oil immersion objective (Nikon) using NIS-Elements (Nikon). During the imaging, culture dishes were kept in a temperature-controlled chamber $\left(37^{\circ} \mathrm{C}\right)$ at $5 \% \mathrm{CO}_{2}$

Structured illumination microscopy. M-L contact sites in both fixed and live neurons were imaged using super-resolution SIM. For SIM analysis, neurons were cultured on nitric-acid-treated, PDL/laminin-coated High Precision Glass Cover Slip (Bioscience Tools, \#CSHP-No1.5-12). Samples were prepared using the same protocol for regular ICC. Super-resolution images were taken on a Nikon N-SIM system with a 100× oil immersion objective lens, 1.49 NA (Nikon). Images were captured and reconstructed using Nikon NIS-Elements.

Neuronal compartmentalization of $\mathbf{M}-\mathbf{L}$ contacts. Dendrites and axons were identified as positive for either MAP2 or Tau immunostaining, respectively, in fixed cells. In live-cell imaging, axons and dendrites were distinguished using Map2-GFP lentiviral expression. Neuron cultures had dendrites that were thicker and shorter than axons, with many axons branching from dendrites, consistent with previous observations in dopaminergic neurons ${ }^{67}$. Using these characteristic morphologies, neurites were further characterized as dendrites in subsequent imaging experiments involving live or fixed neurons.

Image analysis. To quantify contacts, live neurons were imaged at 2-s intervals for 3-6 min. Contacts were defined as mitochondria and lysosomes in close proximity at the beginning of the video and which lasted for greater than $20 \mathrm{~s}$. The duration, number of contacts, and the density of mitochondria were analyzed manually in NIS-Elements (Nikon) for further examination. The minimum duration of contacts was quantified as the time before contact termination and dissociation (mitochondria and lysosomes detaching from one another) over the course of 5-min videos. Any contacts that lasted beyond $5 \mathrm{~min}$ were categorized as $300 \mathrm{~s}$ in bar graphs and as $>300 \mathrm{~s}$ in histograms for the minimum duration of $\mathrm{M}-\mathrm{L}$ contacts. 
The percentage of lysosomes in contacts was quantified as the percentage of lysosomes in contact with mitochondria for greater than $20 \mathrm{~s}$ divided by the total number of Lyso-GFP-positive vesicles in the region of interest. The length of neurites and the area of cell bodies were measured using a built-in function of Image (NIH)

Generation and transduction of lentiviral constructs. Human MAP2 Lentiviral cDNA ORF vector was purchased from Sino Biological Inc. (\#HG13690-ACGLN). Human TBC1D15 ORF in the plasmid generated by Vector Builder was subjected to Q $5^{\circ}$ Site-Directed Mutagenesis (New England Biolabs, \#E0554S) to insert NheI enzyme cutting site (5'-GCTAGC-3) and then cloned into lentiviral vector including BFP tag (Addgene, \#117136). mApple-Tom20 (Addgene, \#54955) and LAMP1-mGFP (Addgene, \#34831) were obtained from Addgene plasmids using the NheI/NotI cut sites and subcloned into pER4 lentiviral expression vector. For TBC1D15 knockdown, shRNA constructs against TBC1D15 were obtained from Horizon Discovery (\#RHS4533-EG64786) together with TRC Lentiviral Nontargeting shRNA Control (\#RHS6848). To generate the lentivirus control, the nuclear localization signal (NLS) of SV40 large T antigen was introduced after BFP coding sequence in the same lentiviral plasmid (Addgene \#117136) used for hTBC1D15 cloning. Lentiviral vectors were packaged and transfected into HEK 293FT cells using X-treme Gene HP DNA transfection (Roche, \#06366236001) together with helper plasmids psPAX2 (Addgene, \#12260) and pLP3 (Invitrogen). Quantitation of retroviral antigens was determined using ZeptoMetrix Corporation RETROtek HIV-1 p24 Antigen ELISA kits (Fisher Scientific, \#22-156-700). Concentrated viruses were aliquoted and kept at $-80^{\circ} \mathrm{C}$ for future use. For Map2 expression, neurons were transduced with Map2 Lentivirus and incubated 4 days before live-cell confocal imaging or fixation. For TBC1D15 expression, neurons at day 35 were transduced with hTBC1D15 Lentivirus, incubated 15 days $(\mathrm{MOI}=8)$ until day 50 and were subjected to further analysis. Expression of constructs delivered by lentiviral infection was verified by either immunoblot analysis or imaging of the fluorescent label.

Live-cell GCase activity assay. The enzymatic activity of GCase in live human neurons was measured as previously described ${ }^{15}$ with minor modifications. The neuron culture media was changed to phenol red-free Neurobasal media (Thermo Fisher, \#12348017) containing Neurocult SM1 (Stemcell Technologies, \#5711) a day before the measurement. The next day, half of the neurons were treated with DMSO and $50 \mu \mathrm{g} / \mathrm{ml}$ fluorescein di- $\beta$-D-glucopyranoside (PFB-FDGluc) (Fisher Scientific, \#P11947) in phenol red-free Neurobasal media. Another half were treated with $400 \mathrm{nM}$ BafAl (Cayman chemicals, \#11038) and $50 \mu \mathrm{g} / \mathrm{ml}$ PFBFDGluc in phenol red-free Neurobasal media. Neurons were incubated for $1 \mathrm{~h}$ at $37^{\circ} \mathrm{C}$ in the dark to allow for the accumulation of PFB-FDGluc, a substrate of GCase, in lysosomes. GCase activity was quantified for $4.5 \mathrm{~h}$ with $30 \mathrm{~min}$ intervals by measuring fluorescence upon the cleavage of PFB-FDGluc over time in a Spectramax i3 plate reader (Molecular Devices) $(\mathrm{Ex}=485 \mathrm{~nm}, \mathrm{Em}=525 \mathrm{~nm}$ ). Activity within the lysosomal compartment was determined by measuring the response to BafA1. Non-lysosomal GCase activity was interpreted as the activity that was not responsive to BafAl. After the last measurement, neurons were washed three times with PBS, and were lysed in RIPA lysis buffer. BCA protein assay was done according to the manufacturer's protocol (Thermo Fisher, \#23225) to measure the total protein amount required for normalization. Total GCase activity was quantified by calculating the area under the DMSO curve (AUC) using Prism7 (GraphPad) software. Lysosomal GCase activity was obtained by measuring the area between BafA1 and DMSO curves.

SDS-PAGE and Western blotting. Neurons were collected in ice-cold PBS and centrifuged at $400 \times g$ for $5 \mathrm{~min}$ at specific time points (e.g. day 30 for neuron characterization, day $40-70$ for biochemical analysis of organelle contacts). Pellets were lysed in N-PER ${ }^{m}$ Neuronal Protein Extraction Reagent (Thermo Scientific, \#87792) with cOmplete protease inhibitor cocktail (Sigma, \#11836170001), and lysates were collected according to the manufacturer's protocol. After boiling for $20 \mathrm{~min}$ in $4 \times$ Laemmeli sample buffer, protein samples were separated on 4-20\% Tris-glycine precasted gel (Invitrogen, \#XP04202BOX) and transferred to PVDF or nitrocellulose membranes using Trans-blot TurboTM transfer system (Biorad). Membranes were blocked with $5 \%$ milk in $1 \times$ Tris-buffered saline $(50 \mathrm{mM}$ Tris, pH 7.4, $150 \mathrm{mM} \mathrm{NaCl})$ with $0.1 \%$ Tween (TBST) for $1 \mathrm{~h}$ at room temperature and incubated with a primary antibody, $4^{\circ} \mathrm{C}$, overnight: Rab7 (Cell Signaling, \#D95F2, 1:1000; Abcam, \#ab137029, 1:1000); TBC1D15 (Sigma Aldrich, \#SAB2701508, 1:500); Fis1 (Alexis, \#ALX-2101037-0100, 1:1000); GAPDH (Millipore, \#MAB374, 1:2000); GBA (Abnova, \#H00002629-M01, 1:500); TH (Calbiochem, \#657012, 1:1000); Synapsin (Santa Cruz, \#sc-398849, 1:1000); $\beta$-III-tubulin (TUJ1) (Biolegend, \#801202, 1:5000); AMPKa (Cell Signaling, \#2532, 1:1000); Phospho-AMPKa (Cell Signaling, \#2535, 1:1000); Ccz1 (Santa Cruz, \#sc-514290, 1:1000); Mon1 (Abcam, \#ab103919, 1:500); Tom20 (Abcam, \#ab56783, 1:1000); Lamp1 (Santa Cruz, \#sc-20011, 1:500). The next day, after three times of washing with $1 \times$ TBST, membranes were incubated in secondary goat antimouse and goat anti-rabbit HRP antibody (Jackson Immuno Research Lab, \#115-035146 , \#111-035-144, 1:10,000) diluted in 5\% milk in $1 \times$ TBST for $1 \mathrm{~h}$ and washed three times with $1 \times$ TBST. HRP signal was developed using Clarity chemiluminescence substrate (Biorad, \#170-5061) or Lumigen ECL Ultra (Lumigen, \#TMA-100), and images were taken on the ChemiDoc XRS+ imaging station (Biorad). Protein levels were normalized against GAPDH. Quantification was done using ImageJ (NIH).

Quantitative RT-PCR. Total RNA was purified from neurons using RNeasy Micro Kit (Qiagen, \#74004) and transcribed to cDNA using the High-Capacity cDNA Reverse Transcription Kit (Thermo Fisher, \#4368814). Quantitative RT-PCR was performed using the 7500 Fast Real-Time PCR system (Applied Biosystems), with ssoAdvanced universal SYBR Green Supermix (BioRad, \#1725271). The following pre-designed primer sets were used: TBC1D15 (PrimerBank ID \#226342866c3) and GAPDH (PrimerBank ID \#378404907c2) (Supplementary Table 1).

GST-RILP pull-down assay. To determine active GTP-bound Rab7 levels in neurons, GST-RILP pull-down assay was performed as previously described ${ }^{25}$ with minor modifications. Plasmids were gifts from Aimee Edinger (Addgene plasmid \#79149). GST-control and GST-fused Rab7 binding domain of RILP protein (nucleotides 658-897) were expressed in BL21 bacteria. Bacteria were collected and lysed in B-PER ${ }^{\text {tw }}$ Bacterial Protein Extraction Reagent (Thermo Scientific, \#78248) with 1,4-Dithiothreitol (DTT, $1 \mathrm{mM})$, EDTA $(1 \mathrm{mM})$, and cOmplete protease inhibitor cocktail (Sigma, \#11836170001). Proteins were purified using preequilibrated $50 \%$ slurry of glutathione-Sepharose $4 \mathrm{~B}$ beads (GE Healthcare) and quantified using the BCA assay. Neurons to be analyzed in the pull-down assay were pelleted and lysed in pull-down buffer (20 mM HEPES, $100 \mathrm{mM} \mathrm{NaCl}, 5 \mathrm{mM}$ $\mathrm{MgCl}_{2}, 1 \%$ TritonX-100, and protease inhibitors). GST-control beads and GSTRILP beads were added to the neuron lysates and the samples were rocked overnight at $4{ }^{\circ} \mathrm{C}$, followed by washing with cold pull-down buffer. Bound proteins were eluted by boiling in $2 \times$ Laemmli sample buffer (Sigma Aldrich, \#S3401) and used for western blot analysis.

Seahorse XF Cell Mito Stress Test. OCR of neurons was analyzed using an XF 24 Extracellular Flux Analyzer (Seahorse Biosciences) according to the manufacturer's protocol; $1.25 \times 10^{5}$ neurons were plated on one well of XF24 cell culture microplates. Four empty wells without neurons were used as background control for temperature-sensitive fluctuations in OCR analysis. Before the assay, culture medium was replaced with Seahorse XF medium (Seahorse Bioscience, \#103575-100) supplemented with $1 \mathrm{mM}$ sodium pyruvate (Corning $\left.{ }^{\oplus}, \# 25-000-\mathrm{CI}\right), 10 \mathrm{mM}$ D-glucose (Sigma Aldrich, \#G8769), and $2 \mathrm{mM}$ glutamine (Gibco, \#25030-081) and incubated for $1 \mathrm{~h}$ in a $\mathrm{CO}_{2}$-free incubator. OCR was measured, after sequential injection of $1 \mu \mathrm{M}$ oligomycin, CCCP, and Antimycin A. After the assay, neurons were lysed and subjected to BCA protein assay (Thermo Fisher, \#23225) for normalization.

Total cellular ATP assay. Total cellular ATP content was measured using the ATPlite kit (PerkinElmer, \#6016943) according to the manufacturer's protocol. Neurons were plated on black 96-well plates (Thermo Fisher, \#237108) at a density of $5 \times 10^{4} /$ well. Neurons were lysed in lysis solution provided in the kit and luminescence was measured in a Spectramax i3 plate reader (Molecular Devices) Neuron lysates were subjected to BCA protein assay (Thermo Fisher, \#23225) for normalization.

Mitochondrial drug treatment. HeLa cells were cultured in Dulbecco's modified Eagle's medium (DMEM) (Gibco, \#11995-065) supplemented with 10\% FBS, $100 \mathrm{U} / \mathrm{ml}$ Penicillin-Streptomycin (Gibco, \#15140-122). A day after passaging, cells were treated for 0,4 , and $8 \mathrm{~h}$ with CCCP $(10 \mu \mathrm{M})$ (Sigma Aldrich, \#C2759), Rotenone $(100 \mathrm{nM})$ (Sigma Aldrich, \#R8875), Antimycin (1 $\mu \mathrm{M})$ (Sigma Aldrich, \#A8674), or Oligomycin $(1 \mu \mathrm{M})$ (Sigma Aldrich, \#O4876). Treated cells were lysed in RIPA buffer (Boston Bioproduct, \#BP-115-5x) with cOmplete protease inhibitor cocktail (Sigma, \#11836170001), and lysates were subjected to BCA protein assay (Thermo Fisher, \#23225) and SDS-PAGE

Statistical analysis. For all statistical tests, cells from $N \geq 3$ independent experiments (biological replicates) per condition were used (see text and figure legends for details). Data were analyzed using unpaired two-tailed Student's $t$-test (for two datasets) or one-way ANOVA with Tukey's post-hoc test (for multiple datasets). Bar graphs presented are in the form of means \pm SEM. Statistics and graphing were performed using Prism 7 (GraphPad) software. Videos and images were processed using NIS-Elements (Nikon) and assembled using ImageJ. All figures were assembled in Adobe Illustrator.

Reporting summary. Further information on research design is available in the Nature Research Reporting Summary linked to this article.

\section{Data availability}

All data that support the findings of this study are included in the manuscript or are available from the authors upon reasonable request. Source data are provided with this paper. 
Received: 4 May 2020; Accepted: 16 February 2021;

Published online: 22 March 2021

\section{References}

1. Wong, Y. C., Kim, S., Peng, W. \& Krainc, D. Regulation and function of mitochondria-lysosome membrane contact sites in cellular homeostasis. Trends Cell Biol. 29, 500-513 (2019).

2. Wong, Y. C., Ysselstein, D. \& Krainc, D. Mitochondria-lysosome contacts regulate mitochondrial fission via RAB7 GTP hydrolysis. Nature 554, 382-386 (2018).

3. Zhang, X. M., Walsh, B., Mitchell, C. A. \& Rowe, T. TBC domain family, member 15 is a novel mammalian Rab GTPase-activating protein with substrate preference for Rab7. Biochem. Biophys. Res. Commun. 335, 154-161 (2005).

4. Yamano, K., Fogel, A. I., Wang, C., van der Bliek, A. M. \& Youle, R. J. Mitochondrial Rab GAPs govern autophagosome biogenesis during mitophagy. Elife 3, e01612 (2014).

5. Peralta, E. R., Martin, B. C. \& Edinger, A. L. Differential effects of TBC1D15 and mammalian Vps39 on Rab7 activation state, lysosomal morphology, and growth factor dependence. J. Biol. Chem. 285, 16814-16821 (2010).

6. Onoue, K. et al. Fis1 acts as a mitochondrial recruitment factor for TBC1D15 that is involved in regulation of mitochondrial morphology. J. Cell Sci. 126, 176-185 (2013).

7. Klein, C. \& Westenberger, A. Genetics of Parkinson's disease. Cold Spring Harb. Perspect. Med. 2, a008888 (2012).

8. Sulzer, D. \& Surmeier, D. J. Neuronal vulnerability, pathogenesis, and Parkinson's disease. Mov. Disord. 28, 41-50 (2013).

9. Burbulla, L. F. et al. Dopamine oxidation mediates mitochondrial and lysosomal dysfunction in Parkinson's disease. Science 357, 1255-1261 (2017).

10. Nguyen, M., Wong, Y. C., Ysselstein, D., Severino, A. \& Krainc, D. Synaptic, mitochondrial, and lysosomal dysfunction in Parkinson's disease. Trends Neurosci. 42, 140-149 (2019).

11. Sidransky, E. et al. Multicenter analysis of glucocerebrosidase mutations in Parkinson's disease. N. Engl. J. Med. 361, 1651-1661 (2009).

12. Sidransky, E. \& Lopez, G. The link between the GBA gene and parkinsonism. Lancet Neurol. 11, 986-998 (2012).

13. Schondorf, D. C. et al. iPSC-derived neurons from GBA1-associated Parkinson's disease patients show autophagic defects and impaired calcium homeostasis. Nat. Commun. 5, 4028 (2014).

14. Mazzulli, J. R. et al. Activation of beta-glucocerebrosidase reduces pathological alpha-synuclein and restores lysosomal function in Parkinson's patient midbrain neurons. J. Neurosci. 36, 7693-7706 (2016).

15. Mazzulli, J. R. et al. Gaucher disease glucocerebrosidase and alpha-synuclein form a bidirectional pathogenic loop in synucleinopathies. Cell 146, 37-52 (2011).

16. Burbulla, L. F. et al. A modulator of wild-type glucocerebrosidase improves pathogenic phenotypes in dopaminergic neuronal models of Parkinson's disease. Sci. Transl. Med. 11, eaau6870 (2019).

17. Ysselstein, D. et al. LRRK2 kinase activity regulates lysosomal glucocerebrosidase in neurons derived from Parkinson's disease patients. Nat. Commun. 10, 5570 (2019).

18. Nguyen, M. \& Krainc, D. LRRK2 phosphorylation of auxilin mediates synaptic defects in dopaminergic neurons from patients with Parkinson's disease. Proc. Natl Acad. Sci. USA 115, 5576-5581 (2018).

19. Gegg, M. E. et al. Glucocerebrosidase deficiency in substantia nigra of parkinson disease brains. Ann. Neurol. 72, 455-463 (2012)

20. Murphy, K. E. et al. Reduced glucocerebrosidase is associated with increased alpha-synuclein in sporadic Parkinson's disease. Brain 137, 834-848 (2014).

21. Kim, M. J., Jeon, S., Burbulla, L. F. \& Krainc, D. Acid ceramidase inhibition ameliorates alpha-synuclein accumulation upon loss of GBA1 function. Hum. Mol. Genet. 27, 1972-1988 (2018).

22. Kriks, S. et al. Dopamine neurons derived from human ES cells efficiently engraft in animal models of Parkinson's disease. Nature 480, 547-551 (2011).

23. Csordas, G. et al. Structural and functional features and significance of the physical linkage between ER and mitochondria. J. Cell Biol. 174, 915-921 (2006).

24. Mazzulli, J. R., Zunke, F., Isacson, O., Studer, L. \& Krainc, D. alpha-Synucleininduced lysosomal dysfunction occurs through disruptions in protein trafficking in human midbrain synucleinopathy models. Proc. Natl Acad. Sci. USA 113, 1931-1936 (2016).

25. Romero Rosales, K., Peralta, E. R., Guenther, G. G., Wong, S. Y. \& Edinger, A. L. Rab7 activation by growth factor withdrawal contributes to the induction of apoptosis. Mol. Biol. Cell 20, 2831-2840 (2009).

26. Cantalupo, G., Alifano, P., Roberti, V., Bruni, C. B. \& Bucci, C. Rab-interacting lysosomal protein (RILP): the Rab7 effector required for transport to lysosomes. EMBO J. 20, 683-693 (2001).
27. Jordens, I. et al. The Rab7 effector protein RILP controls lysosomal transport by inducing the recruitment of dynein-dynactin motors. Curr. Biol. 11, $1680-1685$ (2001)

28. Cleeter, M. W. et al. Glucocerebrosidase inhibition causes mitochondrial dysfunction and free radical damage. Neurochem. Int. 62, 1-7 (2013).

29. Zunke, F. et al. Reversible conformational conversion of alpha-synuclein into toxic assemblies by glucosylceramide. Neuron 97, 92-107.e110 (2018)

30. Wong, Y. C., Peng, W. \& Krainc, D. Lysosomal regulation of intermitochondrial contact fate and motility in Charcot-Marie-Tooth type 2. Dev. Cell 50, 339-354.e334 (2019).

31. Cioni, J. M. et al. Late endosomes act as mRNA translation platforms and sustain mitochondria in axons. Cell 176, 56-72.e15 (2019).

32. Guo, Y. et al. Visualizing intracellular organelle and cytoskeletal interactions at nanoscale resolution on millisecond timescales. Cell 175, 1430-1442.e1417 (2018).

33. Chang, D. et al. A meta-analysis of genome-wide association studies identifies 17 new Parkinson's disease risk loci. Nat. Genet. 49, 1511-1516 (2017).

34. Abeliovich, A. \& Gitler, A. D. Defects in trafficking bridge Parkinson's disease pathology and genetics. Nature 539, 207-216 (2016).

35. Cuddy, L. K. et al. Stress-Induced cellular clearance is mediated by the SNARE protein ykt6 and disrupted by alpha-synuclein. Neuron 104, 869-884.e811 (2019).

36. Giaime, E. et al. Age-dependent dopaminergic neurodegeneration and impairment of the autophagy-lysosomal pathway in LRRK-deficient mice. Neuron 96, 796-807.e796 (2017).

37. Kim, N. C. et al. VCP is essential for mitochondrial quality control by PINK1/ Parkin and this function is impaired by VCP mutations. Neuron 78, 65-80 (2013).

38. Ordonez, D. G., Lee, M. K. \& Feany, M. B. alpha-Synuclein induces mitochondrial dysfunction through spectrin and the actin cytoskeleton. Neuron 97, 108-124.e106 (2018).

39. Pickrell, A. M. \& Youle, R. J. The roles of PINK1, parkin, and mitochondrial fidelity in Parkinson's disease. Neuron 85, 257-273 (2015).

40. Valadas, J. S. et al. ER lipid defects in neuropeptidergic neurons impair sleep patterns in Parkinson's disease. Neuron 98, 1155-1169.e1156 (2018).

41. Bae, E. J. et al. Glucocerebrosidase depletion enhances cell-to-cell transmission of alpha-synuclein. Nat. Commun. 5, 4755 (2014).

42. de la Mata, M. et al. Pharmacological chaperones and coenzyme Q10 treatment improves mutant beta-glucocerebrosidase activity and mitochondrial function in neuronopathic forms of Gaucher disease. Sci. Rep. 5, 10903 (2015).

43. Gegg, M. E. \& Schapira, A. H. Mitochondrial dysfunction associated with glucocerebrosidase deficiency. Neurobiol. Dis. 90, 43-50 (2016).

44. Li, H. et al. Mitochondrial dysfunction and mitophagy defect triggered by heterozygous GBA mutations. Autophagy 15, 113-130 (2019).

45. Osellame, L. D. et al. Mitochondria and quality control defects in a mouse model of Gaucher disease-links to Parkinson's disease. Cell Metab. 17, 941-953 (2013).

46. Schondorf, D. C. et al. The NAD+ precursor nicotinamide riboside rescues mitochondrial defects and neuronal loss in iPSC and fly models of Parkinson's disease. Cell Rep. 23, 2976-2988 (2018).

47. Aflaki, E. et al. Efferocytosis is impaired in Gaucher macrophages. Haematologica 102, 656-665 (2017)

48. Magalhaes, J. et al. Autophagic lysosome reformation dysfunction in glucocerebrosidase deficient cells: relevance to Parkinson disease. Hum. Mol. Genet. 25, 3432-3445 (2016).

49. Helle, S. C. et al. Organization and function of membrane contact sites. Biochim. Biophys. Acta 1833, 2526-2541 (2013).

50. Daniele, T. \& Schiaffino, M. V. Organelle biogenesis and interorganellar connections: better in contact than in isolation. Commun. Integr. Biol. 7 , e29587 (2014).

51. Eisenberg-Bord, M., Shai, N., Schuldiner, M. \& Bohnert, M. A tether is a tether is a tether: tethering at membrane contact sites. Dev. Cell 39, 395-409 (2016).

52. Hirabayashi, Y. et al. ER-mitochondria tethering by PDZD8 regulates $\mathrm{Ca}(2+)$ dynamics in mammalian neurons. Science 358, 623-630 (2017).

53. $\mathrm{Wu}, \mathrm{Y}$. et al. Contacts between the endoplasmic reticulum and other membranes in neurons. Proc. Natl Acad. Sci. USA 114, E4859-E4867 (2017).

54. Allison, R. et al. Defects in ER-endosome contacts impact lysosome function in hereditary spastic paraplegia. J. Cell Biol. 216, 1337-1355 (2017).

55. Chu, B. B. et al. Cholesterol transport through lysosome-peroxisome membrane contacts. Cell 161, 291-306 (2015)

56. Hoglinger, D. et al. NPC1 regulates ER contacts with endocytic organelles to mediate cholesterol egress. Nat. Commun. 10, 4276 (2019).

57. Lee, K. S. et al. Altered ER-mitochondria contact impacts mitochondria calcium homeostasis and contributes to neurodegeneration in vivo in disease models. Proc. Natl Acad. Sci. USA 115, E8844-E8853 (2018).

58. Liao, Y. C. et al. RNA granules hitchhike on lysosomes for long-distance transport, using annexin A11 as a molecular tether. Cell 179, 147-164.e120 (2019). 
59. Lim, C. Y. et al. ER-lysosome contacts enable cholesterol sensing by mTORC1 and drive aberrant growth signalling in Niemann-Pick type C. Nat. Cell Biol. 21, 1206-1218 (2019).

60. Peng, W., Wong, Y. C. \& Krainc, D. Mitochondria-lysosome contacts regulate mitochondrial $\mathrm{Ca}(2+)$ dynamics via lysosomal TRPML1. Proc. Natl Acad. Sci. USA 117, 19266-19275 (2020).

61. Valm, A. M. et al. Applying systems-level spectral imaging and analysis to reveal the organelle interactome. Nature 546, 162-167 (2017).

62. Aston, D. et al. High resolution structural evidence suggests the sarcoplasmic reticulum forms microdomains with acidic stores (lysosomes) in the heart. Sci. Rep. 7, 40620 (2017).

63. Han, Y., Li, M., Qiu, F., Zhang, M. \& Zhang, Y. H. Cell-permeable organic fluorescent probes for live-cell long-term super-resolution imaging reveal lysosome-mitochondrion interactions. Nat. Commun. 8, 1307 (2017).

64. Fermie, J. et al. Single organelle dynamics linked to 3D structure by correlative live-cell imaging and 3D electron microscopy. Traffic 19, 354-369 (2018).

65. Ran, F. A. et al. Genome engineering using the CRISPR-Cas9 system. Nat. Protoc. 8, 2281-2308 (2013).

66. Stauffer, W., Sheng, H. \& Lim, H. N. EzColocalization: an ImageJ plugin for visualizing and measuring colocalization in cells and organisms. Sci. Rep. 8, 15764 (2018).

67. Hausser, M., Stuart, G., Racca, C. \& Sakmann, B. Axonal initiation and active dendritic propagation of action potentials in substantia nigra neurons. Neuron 15, 637-647 (1995).

\section{Acknowledgements}

We thank Lena Burbulla and members of the Krainc laboratory for advice, and the Northwestern University Stem Cell Core Facility for the generation of the iPSC lines. All imaging work was performed at the Northwestern University Center for Advanced Microscopy, supported by NCI CCSG P30 CA060553 awarded to the Robert H. Lurie Comprehensive Cancer Center. This work was supported by NIH/NINDS grants (F32 NS101778, K99 NS109252) and Warren Alpert Distinguished Scholars Fellowship Award to Y.C.W., and NIH/NINDS grants (R01 NS076054 and R37NS096241) to D.K.

\section{Author contributions}

S.K., Y.C.W., and D.K. provided the conceptual framework and designed the experiments. S.K. performed the cell culture, imaging, and analyzed the data. F.G. performed the cell culture and collected the data. S.K., Y.C.W., and D.K. wrote the manuscript. Figures were created by S.K.

\section{Competing interests}

S.K., Y.C.W., and F.G. declare no competing interests. D.K. is a scientific advisor for Prevail Therapeutics and Intellia Therapeutics, as well as a venture partner at Orbimed.

\section{Additional information}

Supplementary information The online version contains supplementary material available at https://doi.org/10.1038/s41467-021-22113-3.

Correspondence and requests for materials should be addressed to D.K.

Peer review information Nature Communications thanks the anonymous reviewer(s) for their contribution to the peer review of this work. Peer reviewer reports are available.

Reprints and permission information is available at http://www.nature.com/reprints

Publisher's note Springer Nature remains neutral with regard to jurisdictional claims in published maps and institutional affiliations.

\section{(c) (i)}

Open Access This article is licensed under a Creative Commons Attribution 4.0 International License, which permits use, sharing, adaptation, distribution and reproduction in any medium or format, as long as you give appropriate credit to the original author(s) and the source, provide a link to the Creative Commons license, and indicate if changes were made. The images or other third party material in this article are included in the article's Creative Commons license, unless indicated otherwise in a credit line to the material. If material is not included in the article's Creative Commons license and your intended use is not permitted by statutory regulation or exceeds the permitted use, you will need to obtain permission directly from the copyright holder. To view a copy of this license, visit http://creativecommons.org/ licenses/by/4.0/.

(C) The Author(s) 2021 